\title{
Confinement, Deconfinement and Freezing in Lattice Yang-Mills Theories with Continuous Time
}

\author{
Christian Borgs \\ Theoretische Physik, ETH-Hönggerberg, CH-8093 Zürich, Switzerland
}

\begin{abstract}
In this paper I analyse lattice Yang-Mills theories with continuous time. After a short discussion of more conceptual questions, such as the existence of a Hamilton operator in the infinite volume limit, I study the phase diagram. The existence of a strong coupling/low temperature confinement phase (which was not proven up to now) is established for arbitrary compact groups, continuous or discrete. For discrete compact groups the deconfinement region decomposes into (at least) two phases, which are distinguished by the behaviour of spatial Wilson loops: a deconfinement phase where spatial Wilson loops still show area law behaviour, and a "freezing" phase with perimeter law behaviour for spatial Wilson loops. The methods to prove these results rely on cluster expansion methods, combined with renormalisation ideas.
\end{abstract}

\section{Introduction}

Lattice gauge theories (LGT) with continuous time have some advantages over LGT defined on a symmetric lattice: First, the theory is defined directly by a Hamiltonian $H=\frac{g^{2}}{2} \Delta+V$, where $V$ is some potential term and $\Delta$ the LaplaceBeltrami operator on the classical configuration space. Therefore the quantum mechanical interpretation is much more direct as for LGT with discrete time, where the Hamiltonian is given rather abstractly as the logarithm of the transfer matrix. Secondly, LGT with continuous time have advantages if one is interested in high temperature effects. This is due to the fact that the inverse temperature $\beta$ is given by the length of the lattice in time direction. Therefore, the temperature on the symmetric lattice can never be chosen higher than the inverse lattice spacing, whereas it can be chosen arbitrarily high for continuous time.

Unfortunately, however, there have been very few rigorous results [1-4] about the phase structure of the theory with continuous time. For the pure Yang-Mills theory, to which I stick in this article, they all concern the higt: temperature 
deconfinement phase [1-3]. But it was not proven, e.g., that the theory shows confinement for strong couplings (up to now, convergence of the strong coupling expansion sketched by Kogut and Susskind [5] has not been established rigorously). The reason for the fact that the corresponding rigorous results for symmetric lattices (see e.g. $[6,7]$ ) could not be proven for continuous times is due to the fact, that the expansions used to prove these results on the symmetric lattice are divergent in the limit of continuous time.

One could hope, however, that an effective theory on a time scale of order unity can still be treated by strong coupling cluster expansion methods. Indeed I will show in this article that the combination of renormalisation ideas and standard cluster expansion methods leads to a convergent strong coupling expansion for the theory with continuous time. One obtains confinement for infinitely heavy quarks, a non-vanishing mass gap and area law behaviour for spatial Wilson loops, provided the coupling constant $g^{2}$ and the inverse temperature $\beta$ are large.

On the other hand it is known [1] that confinement breaks down if $g^{2}<g_{2}^{2}(\beta)$ $=\operatorname{const} \beta^{-1}$. This does not exclude, however, an area law for spatial Wilson loops, as already pointed out in [8]. In fact, it can be proven that spatial Wilson loops do show area law behaviour, provided $g^{2} \beta^{-1} \gg 1$ [3]. Thus, for sufficiently high temperatures $\beta^{-1}$, there is a region $g_{1}^{2}(\beta)<g^{2}<g_{2}^{2}(\beta)$ where the theory shows deconfinement (in the sense of Polyakov [9]) and area law behaviour for spatial Wilson loops.

For discrete groups there should be an additional phase, the so-called "freezing phase," with deconfinement in the sense of Polyakov and perimeter law behaviour for spatial Wilson loops. I will show the existence of this freezing phase in Sect. 4, using a variant of Ginibre's [10] quantum contour expansion (for technical reasons I only treat discrete abelian groups in Sect. 4; discrete non-abelian groups are discussed in Appendix B).

The organisation of this paper is as follows: In the remaining part of this introduction I explain the main ideas for the continuous time strong coupling expansion. In Sect. 1 I define the theory and discuss several conceptual questions, such as the existence of the Hamilton operator $H$ in the infinite volume limit. In Sect. 2 I derive the strong coupling cluster expansion sketched below. Convergence, as well as the resulting physical properties like mass gap or confinement, are proven in Sect. 3. The quantum contour expansion for the freezing phase is derived in Sect. 4. In Sect. 5 I summarize the results of this paper and discuss the resulting phase diagram. Appendix $\mathrm{A}$ is devoted to a compactness argument concerning the existence of the infinite volume limit for arbitrary couplings $g^{2}>0$, Appendix B to the generalisation of quantum contours to nonabelian discrete groups.

In the remaining part of this introduction I want to sketch the main ideas on which the strong coupling expansion is based: Consider a lattice $\Lambda_{\tau}$ with small lattice spacing $\tau$ in time direction ${ }^{1}$. I choose free boundary conditions (b.c.) and the lattice spacing of the space lattice is set to one. The partition function in the box $\Lambda_{\tau}$ is$$
Z=\int \prod_{\langle x y\rangle \subset A_{\tau}} d g_{x y}\left(\exp \left\{\sum_{p \subset A_{\tau}}^{v}\left(\frac{1}{\tau g^{2}} \operatorname{Re} \chi\left(g_{\partial p}\right)-K\left(\tau g^{2}\right)\right)\right\} \exp \left\{\sum_{p \subset A_{\tau}}^{h} \frac{\tau}{g^{2}} \operatorname{Re} \chi\left(g_{\partial p}\right)\right\}\right) \text {. }
$$

\footnotetext{
${ }^{1}$ I take for $\Lambda_{\tau}$ a box of the form $A_{\tau}=\tau\left\{0,1, \ldots, L_{t}^{\prime}\right\} \times \Omega$, where $\Omega \subset Z^{d}$ is the space lattice and $L_{t}^{\prime}$ is some positive integer. For convenience I assume that $\tau^{-1}$ and $L_{t}=\tau L_{t}^{\prime}$ are integers
} 
Here $g^{2}$ is the coupling constant, $\chi(\cdot)$ is the trace in some faithful representation of $G$, and $g_{\hat{\partial} p}$ is the plaquette variable $g_{\hat{\partial} p}=\prod_{\langle x y\rangle \in \hat{\partial} p} g_{x w}$, where the product is ordered around the loop $\partial p$. The sum $\sum^{v}$ goes over vertical ( $=$ time-like) plaquettes in $\Lambda_{\tau}$, the $\operatorname{sum} \sum^{h}$ over horizontal ( = space-like) plaquettes in $\Lambda_{\tau} \cdot d g_{x y}$ is the Haar measure for the gauge field $g_{x y}$ and $K\left(\tau g^{2}\right)$ is the constant ${ }^{2} K\left(\tau g^{2}\right)=\log \int d g \exp \left\{\left(1 / \tau g^{2}\right) \operatorname{Re} \chi(g)\right\}$.

It is clear from the above form that the usual strong coupling expansion is divergent in the time continuum limit $\tau \rightarrow 0$, because the coupling for the time-like plaquettes is $\tau g^{2}$, rather than $g^{2}$. One could hope however, that the effective theory on the block lattice $A_{b l}=\left\{0,1, \ldots, L_{t}\right\} \times \Omega \subset Z^{d+1}$ is again strongly coupled (recall that we took $\tau$ and $L_{t}^{\prime}$ in such a way that $L_{t}=\tau L_{t}^{\prime}$ is an integer).

Let us first consider the simplified model where the coupling $\tau / g^{2}$ for the horizontal plaquettes is set to zero. Since we have chosen free boundary conditions we may fix a gauge by setting $g_{x y}$ to one for all time-like links ( $A_{0} \equiv 0$ gauge). The partition function of the simplified model then becomes

$$
\tilde{Z}=\int \prod_{\langle x v\rangle \subset \Lambda_{\tau}}^{h} d g_{x y} \exp \left\{\sum_{p \subset \Lambda_{\tau}}^{v}\left(\frac{1}{\tau g^{2}} \operatorname{Re} \chi\left(g_{\hat{o} p}\right)-K\left(\tau g^{2}\right)\right)\right\},
$$

where the product goes over horizontal links in $\Lambda_{\tau}$.

To obtain a model on the block lattice $\Lambda_{b l}$ we use a decimation procedure (i.e. we integrate out all variables $g_{x y}$, for which $\langle x y\rangle$ lies not in the block lattice, keeping the variables $g_{x y}$ with $\langle x y\rangle\left\langle\Lambda_{b l}\right.$ fixed). A minute of reflection shows that for the simplified model in $A_{0}=0$ gauge this leads to the following representation of $\tilde{Z}$ on $\Lambda_{b l}$

$$
\tilde{Z}=\int \prod_{\langle x y\rangle \subset A_{b l}}^{h} d g_{x v} \exp \left\{\sum_{p \subset A_{b l}}^{v} \tilde{S}\left(g_{\partial p}\right)\right\},
$$

where $e^{\Im(\cdot)}$ is the $1 / \tau^{\text {th }}$ convolution power of $\exp \left\{\left(1 / \tau g^{2}\right) \operatorname{Re} \chi(\cdot)-K\left(\tau g^{2}\right)\right\}$.

Next we expand $e^{\tilde{s}(\cdot)}$ around 1 .

$$
\exp \left\{\sum_{p \subset A_{b l}^{v}}^{v} \tilde{S}\left(g_{\hat{c} p}\right)\right\}=\prod_{p \in A_{2}^{v}}\left(1+\tilde{\varrho}_{p}\right)=\sum_{P \subset A_{2}^{v}} \prod_{p \in P} \tilde{\varrho}_{p},
$$

where $\Lambda_{2}^{v}$ denotes the set of vertical plaquettes in $\Lambda_{b l}$ and $\tilde{\varrho}_{p}=\exp \left\{\tilde{s}\left(g_{\partial p}\right)\right\}-1$. As usual (see e.g. [7]) one obtains a representation of the model as a hard core interacting polymer system. Here polymers are connected sets $\gamma$ of vertical plaquettes in $\Lambda_{b l}$ and their activity $\tilde{z}(\gamma)$ is

$$
\tilde{z}(\gamma)=\int \prod_{p \in \gamma} \tilde{\varrho}_{p} \prod_{\langle x y\rangle \subset A_{b l}}^{h} d g_{x y} .
$$

Note that in the limit $\tau \rightarrow 0 e^{\tilde{s}(\cdot)}$ becomes the kernel $\exp \left(\frac{g^{2}}{2} \Delta\right)(\cdot)$, where $\Delta$ is the Laplace Beltrami operator on $G$. Since $\exp \left(\frac{g^{2}}{2} \Delta\right)(\cdot)-1$ is small for large $g^{2}, \tilde{z}(\gamma)$ falls off exponentially with the size $|\gamma|$ of $\gamma$ for large $g^{2}$, uniformly in $\tau \rightarrow 0$. Therefore one can apply the technique of Mayer expansions for dilute polymer systems [11]

\footnotetext{
${ }^{2}$ The constant $K\left(\tau g^{2}\right)$, which drops out in expectation values, is introduced to ensure the existence of the time continuum $\operatorname{limit} \lim Z$ of the partition function $Z$ 
to obtain a convergent strong coupling expansion for the simplified model with continuous time.

For the full model we combine the above steps with an expansion in the horizontal plaquettes. Again we start with the change to $A_{0}=0$ gauge. The next step is the expansion in the horizontal plaquettes. This expansion will not be done separately in every single plaquette of the fine lattice, however. Instead we group together certain plaquettes: let $c$ be a cube in the block lattice which is spanned by a horizontal plaquette and a unit lattice vector in time direction (I call such cubes time-like cubes or vertical cubes). Then $\mathbb{P}(c)$ denotes the set of all horizontal plaquettes in $\Lambda_{\tau}$ which lie inside $c$ (including the top and bottom). The last factor in (0.1) can now be written as

$$
\exp \left\{\frac{\tau}{g^{2}} \sum_{p \subset A_{\tau}}^{h} \operatorname{Re} \chi\left(g_{\partial p}\right)\right\}=\prod_{c \in \Lambda_{3}^{v}} e^{s(c)},
$$

where $s(c) \equiv\left(\tau / g^{2}\right) \sum_{p \in \mathbb{P}(c)} \operatorname{Re} \chi\left(g_{\partial p}\right)$ and $\Lambda_{3}^{v}$ denotes the vertical cubes in $\Lambda_{b l}{ }^{3}$. Note that $\mathbb{P}(c)$ contains $1 / \tau$ plaquettes and therefore the supremum norm of $s(c)$ is equal to $\chi(\mathbb{1}) / g^{2}$; it follows that $s(c)$ becomes small (in the supremum norm) for large $g^{2}$, uniformly in $\tau$. It seems therefore reasonable to expand $(0.5)$ around 1 ,

$$
\prod_{c \in \Lambda_{3}^{v}}\left[1+\left(e^{s(c)}-1\right)\right]=\sum_{W \subset A_{3}^{v}} \prod_{c \in W}\left(e^{s(c)}-1\right) .
$$

Inserted into $(0.1)$ we obtain a representation of $Z$ as a sum of terms $Z(W)$, where $W$ is a set of vertical cubes in the block lattice and

$$
Z(W) \equiv \int \prod_{\langle x y\rangle \subset A_{\tau}} d g_{x y}\left(\exp \left\{\sum_{p \subset A_{\tau}}^{v}\left(\frac{1}{\tau g^{2}} \operatorname{Re} \chi\left(g_{\partial p}\right)-K\left(\tau g^{2}\right)\right)\right\} \prod_{c \in W}\left(e^{s(c)}-1\right)\right) .
$$

To each term $Z(W)$ in this sum we now apply the steps "decimation" and "expand $e^{\tilde{s}}$ around 1", with the only difference that the expansion $e^{\tilde{s}}=1+\tilde{\varrho}_{p}$ is only done for those vertical plaquettes $p$ in $\Lambda_{b l}$ which do not lie in the boundary $\partial c$ of a cube $c \in W$ (everything else already got a small factor from the expansion (0.6) and needs no further treatment). I do not want to explain the details in this introduction, but it should be plaubislbe by now that one gets again a representation of the model as a hard core interacting polymer system, where now polymers are built out of plaquettes and cubes in $\Lambda_{b l}$.

For large $g^{2}$ the polymer system is again dilute uniformly in $\tau$ and we obtain the desired convergent strong coupling expansion for the full model. Confinement for infinitely heavy quarks, a mass gap and other interesting properties of the strong coupling phase follow with a little extra work.

Two remarks should be added: First the choice of the $A_{0}=0$ gauge is not essential in any sense. It was only chosen to simplify the exposition of the main ideas. Secondly the expansion in Sect. 2 will differ from the one presented here by the fact that there is no fine lattice $\Lambda_{\tau}$. Instead we work directly in the time continuum, using the operator formulation of the theory. For the purpose of this introduction I started from a fine lattice $\Lambda_{\tau}$ because the derivation of the strong coupling expansion is much more intuitive in this formulation.

\footnotetext{
${ }^{3}$ In fact the plaquettes in the top and bottom of $c$ should get a factor $1 / 2$ in the definition of $s(c)$, because otherwise they are counted twice in $(0.5)$
} 


\section{Lattice Yang-Mills Theories with Continuous Time}

I consider lattice Yang-Mills theories with continuous time, defined on the lattice $\varepsilon Z^{d}$ with lattice spacing $\varepsilon=1 . \Omega \subset Z^{d}$ is the finite box $\left\{x \in Z^{d}:\left|x_{i}\right| \leqq L\right\}$ and $\Omega_{1}, \Omega_{2}, \ldots$ denotes the set of positively oriented links, plaquettes, $\ldots$ in $\Omega$. As gauge group I choose either a finite group $G$ (i.e. $G=Z_{N}$ ) or a compact Lie group [i.e. $G=S U(N)$ or $U(N)]$. The classical configuration space $G^{\Omega_{1}}$ consists of all maps $\mathbf{g}$ from $\Omega_{1}$ into $G:\langle x y\rangle \mapsto g_{x y}$. For a loop $C$ of nearest neighbour (n.n.) pairs in $\Omega$ one defines the loop variable $g_{C}=\prod_{\langle x y\rangle \in C} g_{x y}$, where the product is ordered around $C$; if $\langle x y\rangle$ is positively oriented one sets $g_{x y} \equiv g_{y x}^{-1}$. Finally a gauge transformation is given by a map $\mathbf{h}: \Omega \rightarrow G$; the gauge transformed field $\mathbf{g}^{\mathbf{h}}=\mathbf{h} \mathbf{g h}^{-1}$ is defined as $g_{x y}^{h}=h_{x} g_{x v} \mathbf{h}_{v}^{-1}$ and for a function $\psi$ of the gauge fields one defines $\psi^{\mathbf{h}}(\mathbf{g}) \equiv \psi\left(\mathbf{h}^{-1} \mathbf{g h}\right)$.

Let $L \subset \Omega_{1}$. I denote by $d g_{L}$ the product Haar measure $\prod_{\langle x y\rangle \in L} d g_{x y}$, instead of $d g_{\Omega_{1}}$ I often simply write $d g_{\Omega}$. The quantum mechanical Hilbert space $\mathscr{H}_{\Omega}$ is now defined as the space of square integrable (with respect to $d g_{\Omega}$ ), gauge invariant functions $\psi$ from $G^{\Omega_{1}}$ into the complex numbers $\mathbb{C}$. Note that $\mathscr{H}_{\Omega}$ is an orthogonal subspace of $\tilde{\mathscr{H}}_{\Omega}=L^{2}\left(d g_{\Omega}\right)$ :

$$
\mathscr{H}_{\Omega}=P_{0} \tilde{H}_{\Omega},
$$

where $P_{0}$ denotes the orthogonal projection onto gauge invariant functions

$$
P_{0}=\int \prod_{x \in \Omega} d g_{x} U(\mathbf{h}) \quad(U(\mathbf{h}) \psi)(\mathbf{g})=\psi\left(\mathbf{h}^{-1} \mathbf{g h}\right) .
$$

To define the Hamiltonian $H_{\Omega}$ one first chooses a faithful representation of $G$. Denote by $\chi(\cdot)$ the trace in this representation and by $d(\cdot, \cdot)$ the metric induced by $\chi$ :

$$
d(g, h)=\left\{2 \chi(\mathbb{1})-2 \operatorname{Re} \chi\left(g h^{-1}\right)\right\}^{1 / 2} .
$$
For finite groups $G$ I set $d_{0}=\min _{g \neq \mathbb{1}} d(g, \mathbb{1})$ and define $\Delta_{x y}$ as the discretised Laplace
operator

$$
\left(\Delta_{x y} \psi_{x y}\right)\left(g_{x y}\right):=\sum_{h_{x y}: d\left(h_{x y}, g_{x y}\right)=d_{0}} \frac{\psi_{x y}\left(g_{x y}\right)-\psi_{x y}\left(h_{x y}\right)}{d_{0}^{2}}
$$

and for compact Lie groups I denote by $\Delta_{x y}$ the Laplace Beltrami operator with respect to the Riemannian metric induced by $d$. The Hamiltonian $H_{\Omega}$ is now defined [5] as

$$
H_{\Omega}=H_{\Omega}^{(0)}+V_{\Omega}
$$

with the kinetic term

$$
H_{\Omega}^{(0)}=\frac{g^{2}}{2} \sum_{\langle x y\rangle \in \Omega_{1}} \Delta_{x v}
$$

and the potential

$$
V_{\Omega}=\frac{1}{2 g^{2}} \sum_{p} d\left(\mathbb{1}, g_{\hat{o} p}\right)^{2} .
$$

The kinetic term corresponds to the term $E^{2}$ (the square of the electric field) and the potential to the term $B^{2}$ (the square of the magnetic field) in the Hamiltonian $\frac{1}{2} \int\left(E^{2}+B^{2}\right)$ of the continuum theory. 
Next I introduce the notion of a local observable. One defines the algebra $\mathscr{A}_{\Omega}$ of observables in $\Omega$ as the algebra of all bounded operators $A$ in $\widetilde{\mathscr{H}}_{\Omega}$ which commute with the gauge transformation $U(\mathbf{h})$. For $\Omega \subset \Omega^{\prime} \mathscr{A}_{\Omega}$ can be viewed as a subalgebra of $\mathscr{A}_{\Omega^{\prime}}$. The algebra $\mathscr{A}$ of local observables is defined as

$$
\mathscr{A}=\bigcup_{\Omega} \mathscr{A}_{\Omega},
$$

where the union goes over all finite $\Omega \subset Z^{d}$. I finally define, following a widespread abuse of notation, the support $\operatorname{supp}(A)$ of a local observable $A$ as the set of all links $l \in \Omega_{1}$ for which there is a bounded operator $B_{l}$ in $L^{2}\left(d g_{l}\right)$ that does not commute with $A$. By Schur's lemma an observable $A$ with $L=\operatorname{supp}(A) \subset \Omega_{1}$ can be written as $\mathbb{1} \otimes A^{\prime}$ with an operator $A^{\prime}$ in $\tilde{\mathscr{H}}(L)=L^{2}\left(d g_{L}\right)$ and the unit operator $\mathbb{1}$ in $\tilde{\mathscr{H}}\left(\Omega_{1} \backslash L\right)$. Most of the time I will not distinguish between $A$ and $A^{\prime}$.

For a local observable $A$ one defines the expectation value $\langle A\rangle_{\beta}$ at inverse temperature $\beta$ and the vacuum expectation value $\langle A\rangle$ by

with

$$
\begin{gathered}
\langle A\rangle_{\beta} \equiv \lim _{\Omega \rightarrow Z^{d}}\langle A\rangle_{\beta, \Omega}, \\
\langle A\rangle \equiv \lim _{\substack{\Omega \rightarrow Z^{d} \\
\beta \rightarrow \infty}}\langle A\rangle_{\beta, \Omega},
\end{gathered}
$$

$$
\begin{gathered}
\langle A\rangle_{\beta, \Omega} \equiv Z_{\beta, \Omega}^{-1} \operatorname{Tr}_{\mathscr{H}_{\Omega}} A e^{-\beta H_{\Omega}}, \\
Z_{\beta, \Omega} \equiv \operatorname{Tr}_{\mathscr{H}_{\Omega}} e^{-\beta H_{\Omega}},
\end{gathered}
$$

where the limit $\Omega \rightarrow Z^{d}$ is the limit $L \rightarrow \infty, \Omega=\left\{x \in Z^{d}:\left|x_{i}\right| \leqq L\right\}$.

Remarks. i) Obviously $H_{\Omega}$ commutes with the gauge transformations $U(\mathbf{h})$ and hence with $P_{0}$.

ii) Let $g^{2}>0$ and $\beta>0$. Then $e^{-\beta H_{\Omega}}$ and $P_{0} e^{-\beta H_{\Omega}}$ are positive trace class operators with strictly positive kernel. Furthermore $\left\|e^{-\beta H_{\Omega 2}}\right\|=\left\|P_{0} e^{-\beta H_{\Omega}}\right\|>0$. These facts are proven below.

iii) The finite volume quantities (1.8) and (1.9) are well defined for all $\beta, g^{2}>0$ due to the above remark ii).

iv) For large $g^{2}$ the existence of the limits (1.6) and (1.7) will be shown in Sects. 2 and 3. If the group $G$ is finite one can use standard compactness arguments (see e.g. [12]) to show that for arbitrary $g^{2}>0$ there exists at least a convergent subsequence. For Lie groups the standard argument breaks down, because $\mathscr{A}$ is not separable in this case. The more involved arguments of [13] are however still valid (see Appendix A).

Proof. Only ii) needs to be proven. Obviously $e^{-\beta H_{\Omega}}$ and $P_{0} e^{-\beta H_{\Omega}}$ are positive. $e^{-\beta H_{S 2}^{(0)}}$ is a trace-class operator with strictly positive kernel. Using Hölder's inequality and Trotter's formula, one obtains the corresponding results for $e^{-\beta H_{\Omega}}$, and hence for $P_{0} e^{-\beta H_{\Omega}}$.

The last statement of remark ii) follows by the methods of [14]: Let $\psi$ be an eigenvector of $e^{-\beta H_{\Omega}}$ to the eigenvalue $\left\|e^{-\beta H_{\Omega}}\right\|$. Due to remark i) $\psi$ can be chosen to be an eigenvector of $P_{0}$ and due to the Perron-Frobenius theorem $\psi$ can be chosen strictly positive. But then it is not possible that $P_{0} \psi=0$. Hence $P_{0} \psi=\psi$ and $\left\|P_{0} e^{-\beta H_{\Omega}}\right\|=\left\|e^{-\beta H_{\Omega}}\right\|$. 
I finally recall the definition of the quark-antiquark potential $V_{q \bar{q}}^{\beta}$ at finite $\beta$, as first given by Polyakov in [9]. In the finite volume, $\Omega, V_{q \bar{q}}^{\beta}$ is the free energy difference

$$
V_{q \bar{q}}^{\beta . \Omega}(x, y)=F_{\beta, \Omega}^{q(x) \bar{q}(x)}-F_{\beta, \Omega}
$$

where $F_{\beta, \Omega}$ is the free energy of the system without external quarks

$$
F_{\beta . \Omega}=-\frac{1}{\beta} \log Z_{\beta . \Omega},
$$

and $F_{\beta, \Omega}^{q(x) \bar{q}(y)}$ is the free energy of the system with an external quark $q$ at $x$ and the antiquark $\bar{q}$ at $y$

$$
\begin{gathered}
F_{\beta . \Omega}^{q(x) \bar{q}(y)}=-\frac{1}{\beta} \log Z_{\beta, \Omega}^{q(x) \bar{q}(y)}, \\
Z_{\beta, \Omega}^{q(x) \bar{q}(y)}=\operatorname{Tr}_{\mathscr{H}} \mathscr{q}_{q(x) \bar{q}(y)} e^{-\beta H_{\Omega},} \\
\mathscr{H}_{q(x) \bar{q}(y)}=P_{q(x) \bar{q}(y)} \tilde{H}_{\Omega}, \\
P_{q(x) \bar{q}(y)}=\int \prod_{z} d h_{z} d_{q}^{2} \overline{\chi_{q}\left(h_{x}\right)} \chi_{q}\left(h_{y}\right) U(\mathbf{h}) .
\end{gathered}
$$

$\chi_{q}(\cdot)$ denotes the trace in the respresentation $q$ of $G$ and $d_{q}=\chi_{q}(\mathbb{1})$.

For the rest of this section I will assume that the thermodynamic limit of (1.10) exists and is translation invariant:

$$
V_{q \bar{q}}^{\beta}(x-y) \equiv \lim _{\Omega \rightarrow Z^{d}} V_{q \bar{q}}^{\beta, \Omega}(x, y) .
$$

If $V_{q \bar{q}}^{\beta}(x-y)$ grows linearly with the distance $|x-y|$ as $|x-y| \rightarrow \infty$, one speaks of linear confinement for external (or infinitely heavy) quarks; if $V_{q \bar{q}}^{\beta}(x-y)$ stays bounded for $|x-y| \rightarrow \infty$ one speaks of deconfinement. The (Polyakov-) string tension is defined as

$$
\sigma_{\beta, q}^{(P)}:=\lim _{|x-y| \rightarrow \infty} \frac{1}{|x-y|} V_{q \bar{q}}^{\beta}(x-y) .
$$

Remarks. v) Let $C$ be a loop in the space lattice $\Omega$ and $\chi_{q}(\cdot)$ be the trace in some irreducible representation $q$ of $G$, assumed to represent the center $Z(G)$ of $G$ in a non-trivial way. One often is interested in the question, whether the expectation value $\left\langle\chi_{q}\left(g_{C}\right)\right\rangle_{\beta}$ decays exponentially with the perimeter $|C|$ of $C$ or with the area $A(C)$ of the minimal surface that can be spanned into $C$ (one says: spatial Wilson loops show perimeter respectively area law behaviour). In contrast to the theory on a symmetric space time lattice of zero temperature however, this behaviour is no confinement criterion in the context considered here. In fact spatial Wilson loops probably show area law behaviour for all $\beta<\infty$ and all $g^{2}>0$, provided $d+1 \leqq 4$ and $G$ is continuous [3], whereas $\sigma_{q}$ is known to be zero for $\beta^{-1}>$ const $g^{2}$ in $d+1 \geqq 4$ dimensions [1].

vi) For the theory defined on a space-time lattice the "2-point-function"

$$
G_{\beta, \Omega}(x, y)=\frac{Z_{\beta, \Omega}^{q(x) \bar{q}(y)}}{Z_{\beta, \Omega}} \equiv e^{-\beta \nu_{\mu, \Omega}^{\beta, \Omega(x, y)}}
$$

is nothing else but the expectation value of two Polyakov-loops (also called Wilson-lines in the literature) at distance $|x-y|$, times the trivial multiplicative 
factor $d_{q}^{2}$ (see e.g. [1]). Clearly exponential clustering of the 2-point function (1.18) is equivalent to linear confinement and long range order is equivalent to deconfinement.

Finally I want to discuss the existence of the Hamilton operator $H$ in the infinite volume $Z^{d}$. I first recall the GNS construction of the infinite volume Hilbert space $\mathscr{H}:$ let $\mathscr{N} \equiv\left\{A \in \mathscr{A}:\left\langle A^{*} A\right\rangle=0\right\}$. Then $(A+\mathscr{N}, B+\mathscr{N}) \equiv\left\langle A^{*} B\right\rangle$ defines a positive scaler product on $\mathscr{A} / \mathscr{N}$. $\mathscr{H}$ is the completion of $\mathscr{A} / \mathscr{N}$ with respect to $(\cdot, \cdot)$. For $A \in \mathscr{A}$ I denote by $\Psi_{A}$ the corresponding vector in $\mathscr{H}$. The vector $\Omega \equiv \Psi_{\mathbb{1}}$ is called the vacuum.

For $A, B \in \mathscr{A}$ and for $t \geqq 0$ let

where for $t<\beta$ and $A, B \in \mathscr{A}$,

$$
\omega_{i t}(A, B) \equiv \lim _{\substack{\Omega \rightarrow Z^{d} \\ \beta \rightarrow \infty}} \omega_{i t}^{\beta, \Omega}(A, B),
$$

$$
\omega_{i t}^{\beta, \Omega}(A, B)=Z_{\beta, \Omega}^{-1} \operatorname{Tr}_{\mathscr{H} \Omega} A^{*} e^{-t H_{\Omega}} B e^{-(\beta-t) H_{\Omega}} .
$$

Using the $\log$ convexity of $\omega_{i t}(A, A)$ and the fact that $0 \leqq \omega_{i t}(A, A) \leqq\|A\|^{2}$, one shows the monotonicity of $\omega_{i t}(A, A): \omega_{i t}(A, A) \leqq \omega_{i t^{\prime}}(A, A)$ if $0 \leqq t^{\prime} \leqq t$. We now define an operator $T(t)$ in $\mathscr{H}$ by

$$
\left(\Psi_{A}, T(t) \Psi_{B}\right)=\omega_{i t}(A, B) .
$$

Then obviously $0 \leqq T(t) \leqq 1$ and $T(t+s)=T(t) T(s)$ for all $s, t \geqq 0$. $\Omega$ is an eigenvector of $T(t)$ to the eigenvalue $\|T(t)\|=1$.

Lemma 1.1. Assume that $\lim _{t \rightarrow 0} \omega_{i t}(A, A)=\left\langle A^{*} A\right\rangle \forall A \in \mathscr{A}$. Then there is a selfadjoint operator $H \geqq 0$ on $\mathscr{H}$, such that $H \Omega=0$ and $T(t)=e^{-t H} \forall t \geqq 0$.

Proof. Due to the assumption $(\Psi, T(t) \Psi) \rightarrow(\Psi, \Psi)$ as $t \rightarrow 0$ for all vectors $\Psi$ in a dense subset $D \subset \mathscr{H}$. It follows that

$$
\|T(t) \Psi-\Psi\|^{2}=(\Psi, T(2 t) \Psi)+(\Psi, \Psi)-2(\Psi, T(t) \Psi)
$$

goes to zero for $t \rightarrow 0$. Hence $T(t) \rightarrow \mathbb{1}$ strongly as $t \rightarrow 0$. $H$ is the generator of the strongly continuous semi-group of contractions $\{T(t): t \geqq 0\}[15]$. Since $T(t) \leqq 1$, $H \geqq 0$. $H \Omega=0$ because $T(t) \Omega=\Omega$.

Remarks. vii) For finite groups one can use standard methods (see e.g. [12]) to construct a strongly continuous time evolution $\alpha_{t}(\cdot)$ on $\mathscr{A}$. Since $\omega_{i t}(A, A)$ $=\left\langle A^{*} \alpha_{i t}(A)\right\rangle$ this immediately implies the assumption of the above lemma and hence the existence of the infinite volume hamiltonian $H$.

viii) For general compact groups one can use the expansion of Sect. 2 to show that the assumption of the above lemma holds within the region of convergence of the expansion, i.e. for large $\beta$ and $g^{2}$.

ix) Denote by $\mathscr{H}_{\perp}$ the set of vectors in $\mathscr{H}$ which are orthogonal to $\Omega$. Assume that there is a constant $M>0$ such that $\forall t \geqq 0$ and $\forall A, B \in \mathscr{A}$

$$
\left|\omega_{i t}(A, B)-\left\langle A^{*}\right\rangle\langle B\rangle\right| \leqq \text { const } e^{-M t}
$$

with a constant const which does not depend on $t$. Then, obviously, $H \geqq M$ on $\mathscr{H}_{\perp}$. Hence (1.21) implies a mass gap which is at least $M$. 


\section{The Strong Coupling Cluster Expansion}

In this section I derive an expansion for the time continuum theory which converges for large couplings $g^{2}$ (the corresponding bound $\bar{g}^{2}(\beta)$ depends on the inverse temperature $\beta$ ). Within the region of convergence I show a mass gap, confinement for external quarks and an area law for spatial Wilson loops. The expansion will be explained in this section; convergence and the above mentioned physical consequences will be shown in Sect. 3 .

It is convenient to introduce two coupling constants $\mu, \lambda$ and to consider the expectation values

$$
\langle A\rangle_{N, \Omega}^{\lambda, \mu}=\left(Z_{N, \Omega}^{\lambda, \mu}\right)^{-1} \operatorname{Tr}_{\mathscr{H} \Omega}\left(A T^{N}\right)
$$

where

$$
\begin{gathered}
T=e^{D+W}, \\
D=\sum_{\langle x y\rangle \Omega_{1}} D_{x y}, \quad W=\sum_{p \in \Omega_{2}} W(p), \\
D_{x y}=-\lambda \Delta_{x y}, \quad W(p)=\mu \operatorname{Re}\left(\chi\left(g_{p}\right)-\chi(\mathbb{1})\right) .
\end{gathered}
$$

Here $N$ is a positive integer and $Z_{N, \Omega}^{\lambda, \mu}$ is chosen in such a way that $\langle\mathbb{1}\rangle_{N, \Omega}^{\lambda, \mu}=1$. Clearly $\langle A\rangle_{N, \Omega}^{\lambda, \mu}$ is equal to $\langle A\rangle_{\beta, \Omega}$ as defined in Sect. 1 if $N, \lambda$ and $\mu$ are chosen appropriately. Large $\mathrm{g}^{2}$ correspond to large $\lambda$ and small $\mu$. Throughout this section I will chose $\lambda>0, \mu>0$.

In a first step we rewrite the partition function as an integral involving the kernel $T(\cdot, \cdot)$ of $T$,

$$
\begin{aligned}
Z_{N, \Omega}^{\lambda, \mu} & =\operatorname{Tr}_{\mathscr{H}_{\Omega}}\left(P_{0} T^{N}\right)=\operatorname{Tr}_{\mathscr{H}_{\Omega}}\left(P_{0} T\right)^{N} \\
& =\int \prod_{t=1}^{N} d \mathbf{g}_{t} \prod_{t=1}^{N} d \mathbf{h}_{t} \prod_{t=1}^{N} T\left(\mathbf{h}_{t} \mathbf{g}_{t} \mathbf{h}_{t}^{-1}, \mathbf{g}_{t+1}\right),
\end{aligned}
$$

where for $t=1, \ldots, N \mathbf{g}_{t}$ and $\mathbf{h}_{t}$ are functions from $\Omega_{1}$ and $\Omega$, respectively, into $G$. $d \mathbf{h}_{t}$ and $d \mathbf{g}_{t}$ denote the corresponding product Haar measures; $\mathbf{h}_{t} \mathbf{g}_{t} \mathbf{h}_{t}^{-1}$ is the gauge transformed field.

Next we expand the kernel of $T$ around one. This is done in two steps: first we expand $T$ around $T^{(0)}=e^{D}$ using the Duhamel expansion (a reference concerning the Duhamel expansion is e.g. [16]) and then we expand the kernel $T^{(0)}\left(\mathbf{g}, \mathbf{g}^{\prime}\right)$ around one, using the fact that the heat kernel $e^{-\lambda \Delta_{x y}(\cdot, \cdot)}$ does not differ very much from 1 for large $\lambda$. The first step corresponds to the expansion in the horizontal plaquettes described in the introduction, the second to the expansion in the vertical plaquettes.

The Duhamel expansion gives

$$
e^{D+W}=e^{D+\sum_{p \in \Omega_{2}}^{\sum} W(p)}=\left[\prod_{p \in \Omega_{2}}\left(\sum_{n_{p}=0}^{\infty} \frac{1}{n_{p} !} \int_{0}^{1} d t_{p}^{1} \ldots d t_{p}^{n_{p}}\right)\right] T(\mathbf{t}),
$$

where $T(\mathbf{t})$ is obtained from $T^{(0)}$ by "inserting" $W(p)$ at the times $t_{p}^{1}, \ldots, t_{p}^{n_{p}}$. Formally this can be described as follows: Let $p_{1}, \ldots, p_{K}$ be the plaquettes in $\Omega_{2}$, let $n_{i} \equiv n_{p_{t}}$ and $n=n_{1}+\ldots+n_{K}$. Let

$$
\left(s_{1}, \ldots, s_{n}\right)=\pi\left(t_{p_{1}}^{1}, \ldots, t_{p_{1}}^{n_{1}}, \ldots, t_{p_{K}}^{1}, \ldots, t_{p_{K}}^{n_{K}}\right)
$$


be a permutation of $t_{p_{1}}^{1}, \ldots, t_{p_{K}}^{n_{K}}$ such that $s_{1} \leqq s_{2} \leqq \ldots \leqq s_{n}$ and set

$$
\left(q_{1}, \ldots, q_{n}\right)=\pi\left(p_{1}, \ldots, p_{1}, \ldots, p_{K}, \ldots, p_{K}\right)
$$

where on the right-hand side each $p_{k}$ occurs exactly $n_{k}$ times $(k=1, \ldots, K)$. Then

$$
T(\mathbf{t}) \equiv e^{s_{1} D} W\left(q_{1}\right) e^{\left(s_{2}-s_{1}\right) D} W\left(q_{2}\right) \ldots e^{\left(s_{n}-s_{n-1}\right) D} W\left(q_{n}\right) e^{\left(1-s_{n}\right) D} .
$$

Next we resum (2.6) to obtain

$$
\begin{gathered}
T \equiv e^{D+W}=\sum_{P \subset \Omega_{2}} T(P), \\
T(P)=\left[\prod_{p \in P}\left(\sum_{n_{p}=1}^{\infty} \frac{1}{n_{p} !} \int d t_{p}^{1} \ldots d t_{p}^{n_{p}}\right)\right] T(\mathbf{t}) .
\end{gathered}
$$

To describe the factorization properties of the kernel $T(P)\left(\mathbf{g}, \mathbf{g}^{\prime}\right)$ I need the following definitions: For a set $L \subset \Omega_{1}$ I introduce the operators

$$
\begin{gathered}
D(L)=\sum_{l \in L} D_{l}, \\
T_{L}^{(0)}=e^{D(L)}
\end{gathered}
$$

on $\tilde{\mathscr{H}}(L)=L^{2}\left(d g_{L}\right)$. For $P \subset \Omega_{2}$ let $L(P)$ be the set of links $l$ which belong to the boundary of at least one plaquette in $P$. I then define an operator $\widetilde{T}(P)$ on $\widetilde{\mathscr{H}}(L(P))$ which is obtained from $T_{L(P)}^{(0)}$ in the same way as $T(P)$ is obtained from $T^{(0)}$. More precisely define $T_{L(P)}(\mathbf{t})$ as the right-hand side of $(2.7)$ with $D$ replaced by $D(L(P))$ and $\widetilde{T}(P)$ as the right-hand side of $(2.9)$ with $T(\mathbf{t})$ replaced by $T_{L(P)}(\mathbf{t})$. Obviously the kernel of $T(P)$ has the factorization property

$$
T(P)\left(\mathbf{g}, \mathbf{g}^{\prime}\right)=\prod_{P_{\imath} \in \mathscr{Z}(P)} \tilde{T}\left(P_{i}\right)\left(\mathbf{g}, \mathbf{g}^{\prime}\right) \prod_{L \in L^{c}(P)} T_{l}^{(0)}\left(g_{l}, g_{l}^{\prime}\right),
$$

where $T_{l}^{(0)}=e^{D_{l}}, L^{c}(P)=\Omega_{1} \backslash L(P)$ and $\mathscr{Z}(P)$ denotes the connectivity components of $P$. Note that $\widetilde{T}(P)\left(\mathbf{g}, \mathbf{g}^{\prime}\right)$ depends only on $g_{l}$ and $g_{l}^{\prime}$ if $l \in L(P)$.

In the second step we expand the kernel of $T_{l}^{(0)}$ around 1

$$
T_{l}^{(0)}\left(g_{l}, g_{l}^{\prime}\right)=1+K_{l}\left(g_{l}, g_{l}^{\prime}\right)
$$

provided $l \in L^{c}(P)$. Combined with (2.8) and (2.12) this gives

$$
T=\sum_{P \subset \Omega_{2}} \sum_{L \subset L^{c}(P)} T(P, L)
$$

where $T(P, L)$ is the operator on $\tilde{\mathscr{H}}_{\Omega}$ with kernel

$$
[T(P, L)]\left(\mathbf{g}, \mathbf{g}^{\prime}\right)=\prod_{P_{l} \in \mathscr{\mathscr { C }}(P)} \tilde{T}\left(P_{i}\right)\left(\mathbf{g}, \mathbf{g}^{\prime}\right) \prod_{l \in L} K_{l}\left(g_{l}, g_{l}^{\prime}\right) .
$$

Inserting (2.14)-(2.15) into (2.5) we get a representation of $Z_{N, \Omega}^{\lambda, \mu}$ as the partition function of a polymer system, where polymers are built out of time-like cubes and plaquettes in the space-time lattice $\Lambda=\{0,1, \ldots, N\} \times \Omega$. [The cubes come from the plaquettes in (2.14), the plaquettes from the links in (2.14).] More precisely I define ( $\Lambda$ has to be taken periodic in time direction; $\Lambda_{2}^{v}$ and $\Lambda_{3}^{v}$ denote the positively oriented time-like plaquettes and cubes in $A$; " $v$ " stands for "vertical"): 
Definition 2.1. Let $e, e^{\prime} \in \Lambda_{2}^{v} \cup \Lambda_{3}^{v}$. Then $e$ and $e^{\prime}$ are called incompatible (or connected) if they have at least one common link. For $W \subset \Lambda_{3}^{v} P(W)$ denotes the set of time-like plaquettes $p \in \Lambda_{2}^{v}$ which belong to the boundary of at least one cube $c \in W ; P^{c}(W) \equiv \Lambda_{2}^{v} \backslash P(W)$.

A pair $\gamma=(W, P)$ with $W \subset \Lambda_{3}^{v}, P \subset \Lambda_{2}^{v}$ is called a polymer if $P \subset P^{c}(W)$ and $P \cup W$ is a nonempty connected set. Two polymers $\gamma=(W, P)$ and $\gamma^{\prime}=\left(W^{\prime}, P^{\prime}\right)$ are called incompatible if $W \cup P \cup W^{\prime} \cup P^{\prime}$ is connected (write $\gamma \ngtr \gamma^{\prime}$ ). Otherwise they are called compatible (write $\left.\gamma \sim \gamma^{\prime}\right)$. Finally the size $|\gamma|$ of $\gamma=(W, P)$ is defined as $|W|+|P|$, where $|W|$ and $|P|$ denote the number of cubes and plaquettes in $P$ and $W$, respectively.

For each plaquette $p \in \Lambda_{2}^{v}$ there is exactly one time $t \in\{1, \ldots, N\}$ and one nearest neighbour pair $\langle x y\rangle \in \Omega_{1}$ such that $p=((t, x),(t, y),(t+1, y),(t+1, x))$. I denote $t$ and $\langle x y\rangle$ by $t(p)$ and $\pi(p)$, respectively. For $c \in \Lambda_{3}^{v} t(c) \in\{1, \ldots, N\}$ and $\pi(c) \in \Omega_{2}$ are defined analogously. To define the activity $z(\gamma)$ of a polymer $\gamma=(W, P)$ I decompose $\gamma$ into "time-slices": $\gamma_{1}=\left(W_{1}, P_{1}\right), \ldots, \gamma_{N}=\left(W_{N}, P_{N}\right)$ with $t\left(W_{i}\right)=t\left(P_{i}\right)=i$. I set

$$
\begin{gathered}
\varrho(\gamma)=\prod_{i=1}^{N} \varrho\left(W_{t}\right) \varrho\left(P_{t}\right), \\
\varrho\left(W_{t}\right)=\prod_{P \in \mathscr{X}\left(\pi\left(W_{t}\right)\right)} \tilde{T}(P)\left(\mathbf{h}_{t} \mathbf{g}_{t} \mathbf{h}_{t}^{-1}, \mathbf{g}_{t+1}\right), \\
\varrho\left(P_{t}\right)=\prod_{\langle x y\rangle \in \pi\left(P_{t}\right)} K_{x y}\left(\left(h_{t} g_{t} h_{t}^{-1}\right)_{x y},\left(g_{t+1}\right)_{x y}\right) .
\end{gathered}
$$

We now use (2.14)-(2.18) to rewrite the integrand of (2.5) as

$$
\prod_{t=1}^{N} T\left(\mathbf{h}_{t} \mathbf{g}_{t} \mathbf{h}_{t}^{-1}, \mathbf{g}_{t+1}\right)=\sum_{n=0}^{\infty} \sum_{\left\{\gamma_{1}, \ldots, \gamma_{n}\right\}} \Phi\left(\gamma_{1}, \ldots, \gamma_{n}\right) \prod_{i=1}^{n} \varrho(\gamma ;),
$$

where the sum goes over sets $\left\{\gamma_{1}, \ldots, \gamma_{n}\right\}$ of polymers and $\Phi\left(\gamma_{1}, \ldots, \gamma_{n}\right)=1$ if $\gamma_{1}, \ldots, \gamma_{n}$ are pairwise compatible and $=0$ otherwise. Combined with (2.5) this gives the desired representation of $Z_{N, \Omega}^{\lambda, \mu}$ as the partition function of a polymer system:

$$
Z_{N, \Omega}^{\lambda, \mu}=\sum_{n=0}^{\infty} \sum_{\left\{\gamma_{1}, \ldots, \gamma_{n}\right\}} \Phi\left(\gamma_{1}, \ldots, \gamma_{n}\right) \prod_{i=1}^{n} z\left(\gamma_{i}\right)
$$

with

$$
z(\gamma)=\int \varrho(\gamma) \prod_{t=1}^{n} d \mathbf{g}_{t} \prod_{t=1}^{n} d \mathbf{h}_{t} .
$$

[Note that $\varrho\left(\gamma_{i}\right)$ and $\varrho\left(\gamma_{j}\right)$ have no common variables for $\gamma_{i} \sim \gamma_{j}$.] The Mayer expansion for polymer systems then gives an expansion for the free energy

$$
\log Z_{N, \Omega}^{\lambda, \mu}=\sum_{n=1}^{\infty} \sum_{\gamma_{1}, \ldots, \gamma_{n}} \frac{\Phi_{c}\left(\gamma_{1}, \ldots, \gamma_{n}\right)}{n !} \prod_{i=1}^{n} z\left(\gamma_{i}\right)
$$

where $\Phi_{c}$ is the connected part of $\Phi$ (see e.g. [7] or [17]). Note that this expansion is absolutely convergent, provided the polymer system is dilute, i.e. $|z(\gamma)| \leqq e^{-b|\gamma|}$ with a large constant $b$. We will show such a bound in Sect. 3, using the "trace- 
representation"

$$
z(\gamma)=\operatorname{Tr}_{\tilde{H}_{\Omega}}\left\{P_{0} \prod_{t=1}^{N} T\left(\pi\left(W_{t}\right), \pi\left(P_{t}\right)\right)\right\}
$$

of $z(\gamma)$ [(2.21') follows immediately from (2.21) and (2.15)-(2.18)].

It is not hard to generalize the above steps to obtain an expansion for expectation values. One considers the modified partition function ${ }^{4}$

$$
Z(A)=\operatorname{Tr}_{\mathscr{H}_{\Omega}}(\mathbb{1}+A) T^{N}=\sum_{n=0}^{1} \operatorname{Tr}_{\tilde{H}_{s 2}}\left(P_{0} A^{n} T\right)\left(P_{0} T\right)^{N-1}
$$

and derives again a polymer representation for $Z(A)$. The expectation value of $A$ is obtained by taking only the terms linear in $A$ in the Mayer expansion of $\log Z(A)$.

To obtain the desired polymer representation of $Z(A)$ we apply the same strategy as before. We expand $T$ according to (2.14) and $A T$ according to

$$
A T=\sum_{P \subset \Omega_{2}} \sum_{L \subset L^{c}(P)} A T(P, L),
$$

getting again polymers built out of cubes and plaquettes in $\Lambda$. In addition they carry a label $n$ indicating whether $n=0$ or $n=1$ in the terms on the right-hand side of (2.23).

The factorisation properties of the kernel of $A T(P, L)$ become evident if we write (2.15) as an equation between operators: Let $Q_{l, 0}$ be the operator on $\tilde{\mathscr{H}}_{l}$ $=L^{2}\left(d g_{l}\right)$ with kernel $1,\left(Q_{l, 0}\right.$ is the projector on the "vacuum" of $\left.T_{l}\right), K_{l}=T_{l}-Q_{l .0}$. Then Eqs. (2.15) reads

$$
T(P, L)=\left[\bigotimes_{P_{l} \in Z(P)} \tilde{T}\left(P_{i}\right)\right] \otimes\left[\bigotimes_{l \in L} K_{l}\right] \otimes\left[\bigotimes_{l \in L^{c}(P) \backslash L} Q_{l, 0}\right]
$$

Let $\mathscr{A} \subset \Omega_{1}$ be the support of $A$, let $P_{1}, \ldots, P_{m}$ be those connectivity components of $P$ which are not connected with $\mathscr{A}$, and set $P_{A} \equiv P \backslash\left(P_{1} \cup \ldots \cup P_{m}\right), L_{A} \equiv L \cap \mathscr{A}$. Using (2.25) one immediately gets

$$
[A T(P, L)]\left(\mathbf{g}, \mathbf{g}^{\prime}\right)=\left[A T\left(P_{A}, L_{A}\right)\right]\left(\mathbf{g}, \mathbf{g}^{\prime}\right) \times \prod_{i=1}^{m} \tilde{T}\left(P_{i}\right)\left(\mathbf{g}, \mathbf{g}^{\prime}\right) \prod_{l \in L \backslash L_{A}} K_{l}\left(g_{l}, g_{l}^{\prime}\right) .
$$

Note that the kernel of $A T\left(P_{A}, L_{A}\right)$ depends only on the link variables $g_{l}, g_{l}^{\prime}$ with $l \in \mathscr{A} \cup L\left(P_{A}\right)$, and that of $\widetilde{T}\left(P_{i}\right)$ only on those with $l \in L\left(P_{i}\right)$.

The rest is straightforward. One obtains $Z(A)$ as the partitions function of a polymer system

$$
Z(A)=\sum_{n=0}^{\infty} \sum_{\left\{\gamma_{1}, \ldots, \gamma_{n}\right\}} \Phi\left(\gamma_{1}, \ldots, \gamma_{n}\right) \prod_{i=1}^{n} z_{A}\left(\gamma_{i}\right)
$$

where polymers are now certain 3-tuples $\gamma=(W, P, n)$, with $W \subset \Lambda_{3}^{v}, P \subset \Lambda_{2}^{v}$ and $n \in\{0,1\}$. If $\left(W_{1}, P_{1}\right), \ldots,\left(W_{N}, P_{N}\right)$ are the time-slices of $\gamma$ (defined as before), then

$$
z_{A}(\gamma)=\operatorname{Tr}_{\check{\mathscr{H}}_{\Omega}}\left\{P_{0} A^{n} \prod_{t=1}^{N} T\left(\pi\left(W_{t}\right), \pi\left(P_{t}\right)\right)\right\} .
$$

\footnotetext{
${ }^{4}$ In (2.23) I use the fact, that by definition an observable $A$ commutes with the gauge transformations and hence with $P_{0}$
} 
The precise definition of a polymer and of the notion "compatible" is given in the following Definition 2.2.

Definition 2.2. Let $\mathscr{A}$ be the support of $A$, considered as a subset of $\Lambda_{1}$, i.e. $\mathscr{A}$ consists of the links of the form $(t=1, l) \in \Lambda_{1}$ with $l \in \operatorname{supp}(A)$. I define: A polymer is a 3-tuple $\gamma=(W, P, n)$ with $W \subset \Lambda_{3}^{v}, P \subset \Lambda_{2}^{v}$ and $n \in\{0,1\}$ such that either $n=0$ and $(W, P)$ is a polymer according to Definition 2.1 (in this case I say $\gamma$ is an ordinary polymer and define $\bar{\gamma}:=W \cup P$ ) or $n=1$ and $(W, P)$ is the (possibly empty) union of polymers $\left(W_{1}, P_{1}\right), \ldots,\left(W_{k}, P_{k}\right)$ according to Definition 2.1 , such that for $i=1, \ldots, k$ $W_{i} \cup P_{i}$ is connected with $\mathscr{A}$ (in this case I say $\gamma$ is an A-polymer and define $\bar{\gamma}:=W \cup P \cup \mathscr{A})$.

$\bar{\gamma}$ is called the enlarged support of $\gamma$ and $|\gamma| \equiv|W|+|P|$ the size of $\gamma$. Two polymers $\gamma, \gamma^{\prime}$ are called compatible (write $\gamma \sim \gamma^{\prime}$ ) if $\bar{\gamma}$ and $\bar{\gamma}^{\prime}$ are not connected to each other.

Remark. i) If $\operatorname{supp}(A)$ is not a connected set, $\bar{\gamma}$ is not necessary a connected set. In this case the last definition should be read "if none of connectivity components of $\bar{\gamma}$ is connected to a connectivity component of $\bar{\gamma}^{\prime}$." Note that two $A$-polymers are always incompatible.

From (2.27) we get the expectation value of $A$

$$
\langle A\rangle_{N, \Omega}^{\lambda, \mu}=\sum_{n=1}^{\infty} \sum_{\gamma 1, \ldots, \gamma_{n}}^{\prime} \frac{\Phi_{c}\left(\gamma_{1}, \ldots, \gamma_{n}\right)}{n !} \prod_{i=1}^{n} Z_{A}\left(\gamma_{i}\right),
$$

where the $\sum^{\prime}$ goes over terms linear in $A . \Phi$ and $\Phi_{c}$ are defined as before. Again, this expansion is convergent, provided the polymer system is dilute. Here one needs a bound of the form

$$
\left|z_{A}(\gamma)\right| \leqq\|A\|^{n} e^{-b|\gamma|}
$$

with $b \gg 1$ and a suitable norm on $A$ (we will use the operator norm).

I finally derive a cluster expansion for the quark-antiquark potential, or more precisely for the 2-point-function $G_{\beta, \Omega}=\exp \left(-\beta V_{q \bar{q}}^{\beta, \Omega}\right)$. Again I use the parameters $\lambda, \mu$ and $N$

$$
G_{N, \Omega}^{\lambda, \mu}(x, y)=\left(Z_{N, \Omega}^{\lambda, \mu}\right)^{-1} \operatorname{Tr}_{\mathscr{H}_{\Omega}}\left(P_{q(x) \bar{q}(y)} T\right)^{N} .
$$

The modified partition function is now defined as

$$
\begin{aligned}
Z(\alpha) & \equiv \operatorname{Tr}_{\mathscr{P} \rho_{2}}\left\{\left(P_{0} T\right)^{N}+\alpha\left(P_{q(x) \bar{q}(y)} T\right)^{N}\right\} \\
& =\sum_{n=0,1} \int[\alpha P(x, y)]^{n} \prod_{t=1}^{N} T\left(\mathbf{h}_{t} \mathbf{g}_{t} \mathbf{h}_{t}^{-1}, \mathbf{g}_{t+1}\right) \prod_{t=1}^{N} d \mathbf{g}_{t} \prod_{t=1}^{N} d \mathbf{h}_{t},
\end{aligned}
$$

where

$$
P(x, y)=\prod_{t=1}^{N} d_{q}^{2} \overline{\chi_{q}\left(h_{t x}\right)} \chi_{q}\left(h_{t y}\right) .
$$

The 2-point function is obtained from $Z(\alpha)$ in the same way as $\langle A\rangle$ was obtained from $Z(A)$ :

$$
G_{N, \Omega}^{\lambda, \mu}(x, y)=\left[\frac{d}{d \alpha} \log Z(\alpha)\right]_{\alpha=0} .
$$


I now proceed very similar to the derivation of (2.27): For $z \in \Omega$ let $L_{z}$ be the set of links $\langle(t, z)(t+1, z)\rangle, t=1, \ldots, N$. A polymer is then defined as in Definition 2.2, with the only difference that $L_{x} \cup L_{y}$ plays the role of $\mathscr{A}$. The notion " $A$-polymer" is replaced by "Polyakov-polymer." The activity $z_{\alpha P(x, y)}(\gamma)$ is defined by $\left(2.21^{\prime}\right)$ if $\gamma$ is an ordinary polymer and by

$$
z_{\alpha P(x, y)}(\gamma)=\operatorname{Tr}_{\tilde{\mathscr{H}} \Omega}\left\{\alpha P_{q(x) \bar{q}(y)} \prod_{t=1}^{N} T\left(\pi\left(W_{t}\right), \pi\left(P_{t}\right)\right)\right\}
$$

if $\gamma$ is a Polyakov-polymer with time slices $\left(W_{1}, P_{1}\right), \ldots,\left(W_{N}, P_{N}\right)$. With these definitions one gets

$$
Z(\alpha)=\sum_{n=0}^{\infty} \sum_{\left\{\gamma_{1}, \ldots, \gamma_{n}\right\}} \Phi\left(\gamma_{1}, \ldots, \gamma_{n}\right) \prod_{i=1}^{n} z_{\alpha P(x, y)}\left(\gamma_{i}\right)
$$

and hence

$$
G_{N, \Omega}^{\lambda, \mu}(x, y)=\sum_{n=1}^{\infty} \sum_{\gamma_{1}, \ldots, \gamma_{n}}^{\prime} \frac{\Phi_{c}\left(\gamma_{1}, \ldots, \gamma_{n}\right)}{n !} \prod_{i=1}^{n} z_{P(x, y)}\left(\gamma_{i}\right)
$$

where the sum $\sum^{\prime}$ goes over sequences $\gamma_{1}, \ldots, \gamma_{n}$ containing exactly one Polyakovpolymer. To provve convergence of the expansion (2.35) we will show a bound

$$
\left|z_{P(x, y)}(\gamma)\right| \leqq e^{-b|\gamma|}
$$

both for ordinary and for Polyakov-polymers.

Remarks. ii) The resummation leading to (2.8) can be justified by the fact that (2.6) is convergent in trace norm. This can easily be seen from the inqueality

$$
\operatorname{Tr}_{\mathscr{H}_{\Omega}}|T(\mathbf{t})| \leqq \kappa_{2}^{n} \operatorname{Tr}_{\mathscr{H}_{\Omega}}\left|T^{(0)}\right|
$$

to be proven below. Here $|A|$ denotes the operator $|A|=\left(A^{*} A\right)^{1 / 2}$ and $\kappa_{2}=2 \chi(\mathbb{1})$ $\times|\mu|$. The resummation leading from (2.8) to (2.14) needs no justufication because it is a finite resummation.

iii) Equations (2.5) and (2.21') need a justification, because for a trace class operator $A$ on $\tilde{\mathscr{H}}_{\Omega}$ with kernel $K_{A}$, it is in general not true, that $\operatorname{Tr} A=\int d \mathbf{g} K_{A}(\mathbf{g}, \mathbf{g})$. On the other hand it is well known that the corresponding equality for HilbertSchmidt operators

$$
\operatorname{Tr} A B=\int d \mathbf{g} d \mathbf{g}^{\prime} K_{A}\left(\mathbf{g}, \mathbf{g}^{\prime}\right) K_{B}\left(\mathbf{g}^{\prime}, \mathbf{g}\right)
$$

is true. Therefore (2.5) is well justified for $N \geqq 2$ [take, e.g. $A=P_{0} T$ and $\left.B=\left(P_{0} T\right)^{N-1}\right]$. To prove the equalitity of (2.21) and $\left(2.21^{\prime}\right)$ (assume again $\left.N \geqq 2\right)$ it is obviously enough to show that $T(P, L)$ is Hilbert-Schmidt. This will be done in Sect. 3; in fact we will prove the stronger statement that $T(P, L)$ is trace class [inequality (3.7)-(3.8)].

The case $N=1$ needs some care in the definition of $T(\cdot, \cdot)$, but Eqs. (2.5) and $\left(2.21^{\prime}\right)$ can be justified also in this case. I don't present any details (they are easy!), because the problem can be completely avoided for $N=1$ (see Remark iv)).

iv) If one defines the activity $z(\gamma)$ by $\left(2.21^{\prime}\right)$, the kernels of $T, T(P)$ and $T(P, L)$ only occur in intermediate steps. One could try to derive the polymer representation (2.20) of $Z_{N, \Omega}^{\lambda, \mu}$ without using integral kernels. Clearly this doesn't cause any 
difficulties up to Eq. (2.14) $[T(P, L)$ has to be defined by $(2.25)$ instead of (2.15)]. But after inserting (2.14) into $\operatorname{Tr}\left(P_{0} T\right)^{N}$, we have heavily used the fact that $\varrho\left(\gamma_{i}\right)$ and $\varrho\left(\gamma_{j}\right)$ had no common variables if $\gamma_{i} \sim \gamma_{j}$. And I don't see how the resulting factorisation property could be proven using only (2.25), at least if $N \geqq 2$. For $N=1$ this is easy because now two polymers $\gamma, \gamma^{\prime}$ are connected if and only if their projections onto the space lattice $\Omega$ are connected.

Proof of Remark ii). Let $\alpha_{0}, \ldots, \alpha_{n}$ be positive numbers such that $\alpha_{0}^{-1}$ $+\ldots+\alpha_{n}^{-1}=1$. Then, by Hölder's inequality

$$
\operatorname{Tr}|T(\mathbf{t})| \leqq \prod_{i=1}^{n}\left\|W\left(q_{i}\right)\right\| \prod_{i=0}^{n}\left(\left.\operatorname{Tr}\left|e^{s_{l} D}\right|\right|^{\alpha_{i}}\right)^{\alpha_{i}^{-1}}
$$

Choosing $\alpha_{i}=s_{i}^{-1}$ and bounding $\left\|W\left(q_{i}\right)\right\|$ by $\kappa_{2}$ we get (2.37) (recall that $\left.T^{(0)}=e^{D}\right)$.

\section{Convergence of the Strong Coupling Cluster Expansion}

In this section we will show that the expansion derived in the last section is convergent for large $\lambda$ and small $\mu$, i.e. for large $g^{2}$. With a little extra work we then obtain an area law for horizontal Wilson loops, confinement for external quarks, and exponential clustering for local observables.

Lemma 3.1. i) There is a constant $b_{0}$, depending only on the dimension $d$, such that the cluster expansions (2.29) and (2.35) are absolutely convergent, provided

$$
\begin{gathered}
\left|z_{A}(\gamma)\right| \leqq\left\|A^{n(\gamma)}\right\| e^{-b|\gamma|}, \\
\left|z_{P(x, y)}(\gamma)\right| \leqq e^{-b|\gamma|},
\end{gathered}
$$

with $b>b_{0}$. The convergence of (2.35) is uniform in $\Omega$ and the convergence of (2.29) is uniform in $\Omega$ and $N$.

ii) There are constants $\mu_{0}>0$ and $\lambda_{0}<\infty$, such that (2.30) and (2.36) are true with $b>b_{0}$, provided $\operatorname{Re} \lambda>\lambda_{0}$ and $|\mu|<\mu_{0}$. The constants $\mu_{0}, \lambda_{0}$ only depend on $b_{0}$, the gauge group $G$ and the character $\chi$ used to define the Hamiltonian.

iii) Let $\operatorname{Re} \lambda>0$. Then $z_{A}(\gamma)$ and $z_{P(x, y)}(\gamma)$ are jointly analytic in $\mu$ and $\lambda$.

Proof. i) is standard (see e.g. [7]). The convergence of (2.29) is uniform in $\Omega$ and $N$ because the support of $A$, considered as a subset of the space-time lattice $\Lambda=\{1, \ldots, N\} \times \Omega$ is independent of $\Omega$ and $N$.

ii) We prove ii) using Hölder's inequality, the factorisation property (2.25) and the fact that $\operatorname{Tr}_{\mathscr{H}_{l}}\left|K_{l}\right|$ and $\|W(p)\|$ are bounded by

$$
\begin{gathered}
\omega_{1}=\sum_{q \neq 0} d_{q}^{2} e^{-(\operatorname{Re} \lambda) C_{q}}=\operatorname{Tr}_{\check{\mathscr{H}}_{l} e^{-(\operatorname{Re} \lambda) \Delta_{l}}-1,} \\
\kappa_{2}=2 \chi(\mathbb{1})|\mu|,
\end{gathered}
$$

respectively. Here $C_{q}$ is the quadratic Casimir operator in the irreducible representation $q, d_{q}=\chi_{q}(\mathbb{1})$ and 0 denotes the trivial representation.

We first prove the bound (3.1) [(3.2) needs no proof] and then use (3.1) and (3.2) to show ii). Let $Q_{l, q}$ be the operator on $\tilde{\mathscr{H}}_{l}$ with kernel

$$
Q_{l, q}\left(g_{l}, h_{l}\right)=d_{q}^{2} \chi_{q}\left(g_{l}^{-1} h_{l}\right) \text {. }
$$


Then, by Fourier expanding the kernel of $T_{l}$

$$
T_{l}=\sum_{q} e^{-\lambda C_{q}} Q_{l, q}
$$

and hence

$$
K_{l}=\sum_{q \neq 0} e^{-\lambda C_{q}} Q_{l, q}
$$

Equation (3.4), and the observation that $\operatorname{Tr}\left|Q_{l, q}\right|=\operatorname{Tr} Q_{l, q}=d_{q}^{2}$ immediately imply the bound (3.1).

To bound the left-hand side of (2.30) and (2.36) we use the definitions $\left(2.21^{\prime}\right)$, (2.28) and (2.34) and Hölder's inequality. Since $\left\|P_{0}\right\|=\left\|P_{q(x) \bar{q}(y)}\right\|=1$, we get

$$
\begin{gathered}
\left|z_{A}(\gamma)\right| \leqq\left\|A^{n(\gamma)}\right\| \prod_{t=1}^{N} \operatorname{Tr}_{\tilde{\mathscr{H}}_{\Omega}}\left|T\left(\pi\left(W_{t}\right), \pi\left(P_{t}\right)\right)\right|, \\
\left|z_{p(x, y)}(\gamma)\right| \leqq \prod_{t=1}^{N} \operatorname{Tr}_{\tilde{\mathscr{H}}_{\Omega}}\left|T\left(\pi\left(W_{t}\right), \pi\left(P_{t}\right)\right)\right|
\end{gathered}
$$

if $\gamma$ is a polymer with time slices $\left(W_{1}, P_{1}\right), \ldots,\left(W_{N}, P_{N}\right)$.

Next we use the factorisation formula (2.25), the bound (3.1) and the fact that $\operatorname{Tr} Q_{l, 0}=1$ to obtain the inequality

$$
\begin{aligned}
\operatorname{Tr}_{\tilde{\mathscr{H}} \Omega}|T(P, L)| & =\prod_{l \in L} \operatorname{Tr}\left|K_{l}\right| \prod_{P_{\imath} \in \mathscr{\mathscr { X }}(P)} \operatorname{Tr}_{\tilde{\mathscr{H}}\left(L\left(P_{l}\right)\right)}\left|\widetilde{T}\left(P_{i}\right)\right| \\
& \leqq \omega_{1}^{|L|} \prod_{P_{\imath} \in \mathscr{\mathscr { Z }}(P)} \operatorname{Tr}_{\tilde{\mathscr{H}}\left(L\left(P_{\imath}\right)\right)}\left|\widetilde{T}\left(P_{i}\right)\right| .
\end{aligned}
$$

Recall that $\widetilde{T}(P)$ was defined as a sum over integrals of certain operators $T_{L(P)}(\mathbf{t})$. With the help of Hölder's inequality (cf. Sect. 2, proof of Remark i)) the bound (3.2) implies that

$$
\operatorname{Tr}_{\tilde{\mathscr{H}}(L(P))}\left|T_{L(P)}(\mathbf{t})\right| \leqq\left(\prod_{p \in P} \kappa_{2}^{n(p)}\right) \operatorname{Tr}_{\tilde{\mathscr{H}}(L(P))}\left|T_{L(P)}^{(0)}\right|=\prod_{p \in P} \kappa_{2}^{n(p)} \prod_{l \in L(P)} \operatorname{Tr}\left|T_{l}^{(0)}\right|
$$

and hence (we set $\omega_{2}=\sum_{n \neq 0} \kappa_{2}^{n}$ and bound $\operatorname{Tr}\left|T_{l}^{(0)}\right|=\operatorname{Tr}\left|Q_{l, 0}+K_{l}\right|$ by $\left.\left(1+\omega_{1}\right)\right)$,

$$
\operatorname{Tr}_{\tilde{\mathscr{H}}(L(P))}|\tilde{T}(P)| \leqq \omega_{2}^{|P|}\left(1+\omega_{1}\right)^{|L(P)|}
$$

Equations (3.5)-(3.8), together with the observation that $|\gamma|=\sum\left|\pi\left(W_{i}\right)\right|+\sum\left|\pi\left(P_{i}\right)\right|$ and $|L(P)| \leqq 4|P|$, give the bounds (2.30) and (2.36), with

$$
e^{-b}=\max \left\{\omega_{1}, \omega_{2}\left(1+\omega_{1}\right)^{4}\right\} \text {. }
$$

This completes the proof of ii), because $\omega_{1}$ and $\omega_{2}$ go to zero, if $|\mu| \rightarrow 0$ and $\operatorname{Re} \lambda \rightarrow \infty$, respectively.

iii) Let $\operatorname{Re} \lambda>0$. Then $T^{(0)}$ is trace class and $z_{A}(\gamma)$ can be written as an absolutely convergent sum of integrals $\int d \mathbf{t} \ldots$, involving integrands which are the trace of products of operators $A, P_{0}, W(p)$ and $e^{-t D}$. Using the basis

$$
\mathscr{B}=\bigotimes_{l \in \Omega_{1}} \mathscr{B}_{l}, \quad \mathscr{B}_{l}=\left\{\Psi_{l} \in \widetilde{\mathscr{H}}_{l}: \Psi_{l}=d_{q}^{1 / 2} U_{q}^{a b}(\cdot)\right\}
$$

of $\tilde{\mathscr{H}}_{\Omega}$, where $U_{q}^{a b}(\cdot)$ denotes the matrix elements in the irreducible representation $q$ of $G$, we obtain $z_{A}(\gamma)$ as a sum of integrals $\int d \mathbf{t} \ldots$, involving integrands which are 
sums of finite products of matrix elements,

$$
\left\langle\Psi, A \Psi^{\prime}\right\rangle,\left\langle\Psi, P_{0} \Psi^{\prime}\right\rangle,\left\langle\Psi, W(p) \Psi^{\prime}\right\rangle \text {, and }\left\langle\Psi, e^{-t D} \Psi\right\rangle \text {, }
$$

$\Psi, \Psi^{\prime} \in B$. Discretising integrals and truncating sums we get $z_{A}(\gamma)$ as a limit of functions, which are obviously jointly analytic in $\mu$ and $\lambda$ in the region $\mathscr{D}=\{(\mu, \lambda)$ : $\operatorname{Re} \lambda>0\}$. Let now $K$ be a compact subset of $\mathscr{D}$. By bounds very similar to those used to prove ii) one shows that the approximants to $z_{A}(\gamma)$ are uniformly bounded in $K$. Therefore by Montel's theorem [21], the approximants converge uniformly in any compact subset of $\mathscr{D}$. By Weierstrass's theorem it follows that $z_{A}(\gamma)$ is analytic in the region $\mathscr{D}$.

We now address the question, whether, given $\beta$ and $g^{2}$, we can choose $N, \mu$ and $\lambda$ in such a way that $|\mu|<\mu_{0}, \operatorname{Re} \lambda>\lambda_{0}$ and

$$
\langle A\rangle_{\beta, \Omega}=\langle A\rangle_{N, \Omega}^{\lambda, \mu} \quad \text { and } \quad G_{\beta, \Omega}(x, y)=G_{N, \Omega}^{\lambda, \mu}(x, y) .
$$

The answer is given by the following

Lemma 3.2. Let $g_{0}^{2} \equiv\left(4 \lambda_{0} / \mu_{0}\right)^{1 / 2}, \beta_{0}=\left(\lambda_{0} \mu_{0}\right)^{1 / 2}$ and

$$
\bar{g}^{2}(\beta) \equiv g_{0}^{2} \max \left\{1, \beta_{0} / \beta\right\} .
$$

Then, for $\beta>0$ and $\operatorname{Reg}^{2}>\bar{g}^{2}(\beta), N, \mu$, and $\lambda$ can be chosen in such a way that (3.19) holds and $|\mu|<\mu_{0}, \operatorname{Re} \lambda>\lambda$.

Proof. Choose $N=N(\beta) \equiv \min \left\{N: 2 N>\beta / \beta_{0}\right\} \quad$ and $\quad \mu=\mu_{N}\left\{\beta, 1 / g^{2}\right) \equiv \beta / N g^{2}$, $\lambda=\lambda_{N}\left(\beta, 1 / g^{2}\right) \equiv \beta g^{2} / 2 N$.

The following theorem is an immediate consequence of Lemmas 3.1 and 3.2. $t_{x}(\cdot)$ denotes translation by $x \in \Omega$.

Theorem 3.3. i) Let $\beta>0$ and $\operatorname{Re} g^{2}>\bar{g}^{2}(\beta)$. Then the infinite volume limit

$$
\langle A\rangle_{\beta}=\lim _{\Omega \rightarrow Z^{d}}\langle A\rangle_{\beta, \Omega}
$$

exists for all local observables $A \in \mathscr{A} .\langle\cdot\rangle_{\beta}$ is translation invariant and $\langle A\rangle_{B}$ is an analytic function of $1 / g^{2}$ in the region $\mathscr{G}(\beta)=\left\{1 / g^{2}: 1 / g^{2} \neq 0, \operatorname{Re}^{2}>\bar{g}^{2}(\beta)\right\}$ (see Fig. 1).

ii) For $\beta>0, \operatorname{Reg} g^{2}>\bar{g}^{2}(\beta)$ and $A, B \in \mathscr{A}$ there are constants $K_{A B}<\infty$ and $M>0$, such that

$$
\left|\left\langle A t_{x}(B)\right\rangle_{\beta}-\langle A\rangle_{\beta}\langle B\rangle_{\beta}\right| \leqq K_{A B} e^{-M|x|}
$$

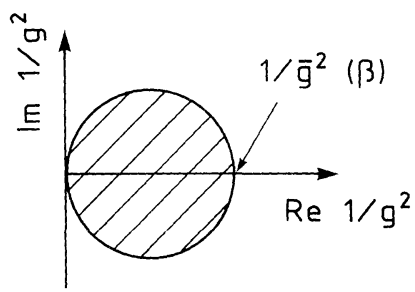

Fig. 1. The region $\mathscr{G}(\beta)$ according to Theorem 3.3 
$\forall x \in \Omega$ (exponential clustering in the spatial directions). $M>0$ can be chosen independently of $A$ and $B$.

Proof. Theorem 3.3 follows from Lemma 3.1 and 3.2 by standard methods. The analyticity of $\langle A\rangle_{\beta}$ follows from the analyticity of $\langle A\rangle_{N}^{\lambda, \mu}=\lim _{\Omega \rightarrow Z^{d}}\langle A\rangle_{N, \Omega}^{\lambda, \mu}$ and the fact that $\mu_{N}$ and $\lambda_{N}$ are analytic functions of $1 / g^{2}$ in the region $\mathscr{G}(\beta)$.

Theorem 3.4. i) Let $\operatorname{Re} g^{2}>g_{0}^{2}$. Then the "full" thermodynamic limit

$$
\langle A\rangle=\lim _{\substack{\Omega \rightarrow Z^{d} \\ \beta \rightarrow \infty}}\langle A\rangle_{\beta, \Omega}
$$

exists $\forall A \in \mathscr{A} .\langle\cdot\rangle$ is translation invariant and $\langle A\rangle$ can be continued to an analytic function of $1 / g^{4}$ in the region $\mathscr{G}=\left\{1 / g^{4}:\left|1 / g^{4}\right|<1 / g_{0}^{4}\right\}$.

ii) For $\operatorname{Reg}^{2}>g_{0}^{2}$ and $A, B \in \mathscr{A}$ there are constants $K_{A B}<\infty$ and $M>0$, such that

$$
\begin{aligned}
& \left|\left\langle A t_{x}(B)\right\rangle-\langle A\rangle\langle B\rangle\right| \leqq K_{A B} e^{-M|x|}, \\
& \left|\omega_{i t}(A, B)-\left\langle A^{*}\right\rangle\langle B\rangle\right| \leqq K_{A B} e^{-M t},
\end{aligned}
$$

$\forall x \in \Omega$ and $\forall t \geqq 0$, respectively (for the definition of $\omega_{i t}$ see (1.19), Sect. 1). Again $M>0$ can be chosen independently of $A$ and $B$.

Proof. The proof of Theorem 3.4 is again fairly standard. The only statement that needs an explanation is that concerning the analyticity properties of $\langle A\rangle$. We first assume that $g_{0}^{2}<g^{2}<\infty$. Then

$$
\langle A\rangle=\lim _{\beta \rightarrow \infty}\langle A\rangle_{\beta}=\lim _{N \rightarrow \infty}\langle A\rangle_{\beta=4 N \lambda_{0} / g^{2}}=\lim _{N \rightarrow \infty}\langle A\rangle_{N}^{\lambda=2 \lambda_{0}, \mu=4 \lambda_{0} / g^{4}} .
$$

So we have shown that for real $g^{2}>g_{0}^{2}\langle A\rangle$ is equal to $f_{A}\left(1 / g^{4}\right)$, where $f_{A}$ is the function

$$
f_{A}(z)=\lim _{N \rightarrow \infty}\langle A\rangle_{N}^{\lambda=2 \lambda_{0}, \mu=4 \lambda_{0} z} .
$$

Due to Lemma 3.1 (note that $\left.\mu_{0}=4 \lambda_{0} / g_{0}^{4}\right) f_{A}$ is analytic in the circle $|z|<1 / g_{0}^{4} . f_{A}$ is the desired analytic continuation of $\langle A\rangle$.

Remarks. i) It is left as an exercise to the reader to derive an expansion for $\omega_{i t}(A, A)$ which is convergent for $\operatorname{Re} g^{2}>g_{0}^{2}$, uniform in $t \in\left[0, t_{0}\right]$ for any $t_{0}<\infty$ (choose the cylinder $\left[0, t_{0}\right] \times \operatorname{supp} \mathscr{A}$ as the set, which replaces $\mathscr{A}$ in Definition 2.2). One immediately obtains that $\omega_{i t}(A, A) \rightarrow\left\langle A^{*} A\right\rangle$ as $t \rightarrow 0$ and hence, by Lemma 1.1, the existence of the infinite volume Hamiltonian $H \geqq 0$.

ii) As explained in Sect. 1 the bound (3.22) implies a mass gap for $H$ which is at least $M$.

iii) The analyticity property of $\langle A\rangle$ is due to the fact that the theory with Hamiltonian

$$
H_{\Omega}^{\prime}=\frac{1}{2} \sum_{\langle x y\rangle \in \Omega_{1}} \Delta_{x y}+\frac{1}{g^{2}} V=\frac{1}{g^{2}} H_{\Omega}
$$

has the same vacuum as the theory with Hamiltonian $H_{\Omega}=\frac{g^{2}}{2} \sum \Delta_{x y}+V$. 
To show confinement for infinitely heavy quarks we need the following lemma [recall that for $x \in \Omega, L_{x}$ denotes the Polyakov loop passing through $(0, x) \in \Lambda$; its consists of the links $\langle(t, x),(t+1, x)\rangle, t=1, \ldots, N]$.

Lemma 3.5. i) Let $\gamma=(W, P, n)$ be a Polyakov polymer with non-vanishing activity. Then each time slice of $\gamma$ contains a connected subset, which connects $L_{x}$ and $L_{y}$, provided the representation $q$ used to define $z_{P(x, y)}(\gamma)$ represents the center of $G$ in $a$ non-trivial way.

ii) Under the above conditions $|\gamma|=|W|+|P| \geqq N \times|x-y|$.

Proof. If $\gamma$ has time slices $\left(W_{1}, P_{1}\right), \ldots,\left(W_{N}, P_{N}\right)$, then

$$
z_{P(x, y)}(\gamma)=\operatorname{Tr}_{\tilde{H}_{\Omega}}\left\{\prod_{t=1}^{N} P_{q(x) \bar{q}(y)} T\left(\pi\left(W_{t}\right), \pi\left(P_{t}\right)\right)\right\} .
$$

It therefore is enough to show that for $P \subset \Omega_{2}, L \subset L^{c}(P)$,

$$
P_{q(x) \bar{q}(y)} T(P, L)=0,
$$

if $L \cup L(P)$ does not contain a path $\omega$ which connects $x$ and $y$.

I now assume that $L \cup L(P)$ does not contain such a path. Let $\mathscr{B}$ be the set of vectors

$$
\Psi_{\{a, b, Q\}} \in \widetilde{\mathscr{H}}_{\Omega}, \quad \Psi_{\{a, b, Q\}}(\mathbf{g})=\prod_{l \in \Omega_{1}} U_{Q(l)}^{a(l) b(l)}\left(g_{l}\right),
$$

where $Q(l)$ denotes an irreducible representation of $G$. Since $\mathscr{B}$ is a base of $\tilde{\mathscr{H}}_{\Omega}$, it is enough to show that

$$
P_{q(x) \bar{q}(y)} T(P, L) \Psi_{\{a, b, Q\}}=0 .
$$

Without loss assume that $\operatorname{supp} Q \subset L \cup L(P)$ (otherwise $T(P, L) \Psi_{\{a, b, Q\}}=0$ ). Since $T(P, L)$ commutes with gauge transformations, it is enough to show that

$$
P_{q(x) \bar{q}(y)} \Psi_{\{a, b, Q\}}=0 .
$$

Assume that (3.23) is false. Then obviously

$$
\left(d^{*} \hat{Q}\right)(z)=\left\{\begin{array}{rl}
\hat{q} & \text { if } z=x \\
-\hat{q} & \text { if } z=y \\
0 & \text { otherwise }
\end{array},\right.
$$

where for an irreducible representation $Q$ of $G \hat{Q}$ denotes the corresponding representation of the center $Z(G)^{5}$. Equation (3.24), however, implies that supp $\hat{Q}$, contains a path connecting $x$ and $y$, which is in contradiction to our assumption that $L \cup L(P) \supset \operatorname{supp} Q \supset \operatorname{supp} \hat{Q}$ contains no path connecting $x$ and $y$. Therefore (3.23) must be true.

ii) follows immediately from i).

The following theorem is a direct consequence of Lemma 3.1, 3.2, and 3.5.

\footnotetext{
${ }^{5}$ We have written $\hat{Z}(G)$, the dual of $Z(G)$, as an additive group; $d^{*}$ denotes the lattice divergence, i.e.

$$
\left(d^{*} \varphi\right)(x)=\sum_{y:\langle x y\rangle \in \Omega_{1}} \varphi(\langle x y\rangle)
$$
}


Theorem 3.6. i) Let $\beta>0$ and $\operatorname{Re} g^{2}>\bar{g}^{2}(\beta)$. Then the infinite volume limit

$$
G_{q \bar{q}}^{\beta}(x, y)=\lim _{\Omega \rightarrow Z^{d}} e^{-\beta V_{q \bar{q}}^{\beta}, \Omega(x, y)}
$$

exists. It is translation invariant and, as a function of $1 / \mathrm{g}^{2}$, analytic in the region $\mathscr{G}(\beta)$ (see Fig. 1).

ii) For representations $q$ which represent the center $Z(G)$ in a non-trivial way, and for $\beta>0, \operatorname{Re}^{2}>\bar{g}^{2}(\beta)$, there are constants $K<\infty, \tilde{\alpha}>0$ such that

$$
\left|G_{q \tilde{q}}^{\beta}(x, y)\right| \leqq K e^{-\tilde{\alpha}|x-y|}
$$

$\forall x, y \in \Omega$.

iii) The constant $\tilde{\alpha}$ in ii) can be chosen in such a way that $\alpha \equiv \tilde{\alpha} / \beta$ stays bounded away from zero in the limit $\beta \rightarrow \infty$.

Remarks. iv) The statement ii) clearly implies confinement for infinitely heavy quarks.

v) Statement iii) shows that the string tension $\sigma_{q, \beta}^{(P)}$ stays bounded away from zero in the limit $\beta \rightarrow \infty$ (zero temperature).

Proof of Theorem 3.6. i) immediately follows from Lemma 3.1 and 3.2

ii) For a power series $\sum a_{n} x^{n}$ with leading power $n_{0}$ and radius of convergence $r_{0}$

$$
\left|\sum a_{n} x^{n}\right| \leqq e^{-n_{0} M} \sum\left|a_{n} x^{n}\right| e^{n M} \leqq \text { const } e^{-n_{0} M}
$$

if $|x| e^{M}<r_{0}$. By the same argument one concludes from Lemma 3.1 and 3.5 that, given $q, \mu$ and $\lambda$ with $q$ as specified in ii) and $|\mu|<\mu_{0}, \operatorname{Re} \lambda>\lambda_{0}$, there is a constant $M>0$ such that

$$
\left|\lim _{\Omega \rightarrow Z^{d}} G_{N, \Omega}^{\lambda, \mu}(x, y)\right| \leqq e^{-N|x-y| \cdot M} .
$$

Together with Lemma 3.2 this immediately implies ii), with $\tilde{\alpha}=N \cdot M$.

iii) We choose $N=N(\beta)$ as in the proof of Lemma 3.2. Then $N(\beta) / \beta$, and hence $\tilde{\alpha} / \beta$, stays bounded away from zero as $\beta \rightarrow \infty$.

I close this section with the proof of the following Theorem $3.7\left(\chi_{q}(\cdot)\right.$ denotes the trace in the irreducible representation $q$ ).

Theorem 3.7. $\forall \beta>0 \forall g^{2}$ with $\operatorname{Re} g^{2}>\bar{g}_{0}^{2}(\beta)$ there are constants $K<\infty, \alpha>0$ such that

$$
\left|\left\langle\chi_{q}\left(g_{C}\right)\right\rangle_{\beta}\right| \leqq K e^{-\alpha|A(C)|}
$$

("area law for spatial Wilson loops"), provided q fulfills the condition stated in Theorem 3.6, ii), and $C$ is selfavoiding $(|A(C)|$ denotes the number of plaquettes in the minimal surface $A(C)$ with boundary $C)$ ) $K$ and $\alpha$ can be chosen in such a way that $K$ stays finite and $\alpha$ stays bounded away from zero in the limit $\beta \rightarrow \infty$.

Proof. Let $\omega: \Omega_{1} \rightarrow G$ be a gauge field which takes values in the center of $G$. Let $P \subset \Omega_{2}$ be a set of plaquettes such that $\omega_{\partial p}=1$ for $p \in P$. Then

$$
\widetilde{T}(P)\left(\boldsymbol{\omega g}, \omega^{\prime}\right)=\widetilde{T}(P)\left(\mathbf{g}, \mathbf{g}^{\prime}\right)
$$

[use the fact that $e^{s D(L)}\left(\boldsymbol{\omega g}, \boldsymbol{\omega g}^{\prime}\right)=e^{s D(L)}\left(\mathbf{g}, \mathbf{g}^{\prime}\right)$ and that $W_{p}(\mathbf{g})=W_{p}(\boldsymbol{\omega g})$ if $p \in P$ ]. 
I now consider a polymer $\gamma=(W, P, n)$ with $n=1$ (I call such polymers Wilson polymers in the sequel). Due to the invariance property (3.27) its activity is zero if it is possible to choose a co-closed set $T \subset \Omega_{2} \backslash P$ such that $C$ has winding number one with respect to $T$ (The proof is left to the reader, see also [19]). It follows that only Wilson polymers $\gamma$ with $|\gamma| \geqq|A(C)|$ contribute to the cluster expansion of $\left\langle\chi_{q}\left(g_{C}\right)\right\rangle_{\beta}$. This immediately implies the theorem.

\section{Deconfinement vs. Freezing for Discrete Groups}

In the last section we have shown confinement for infinitely heavy quarks and an area law for spatial Wilson loops for strongly coupled lattice Yang-Mills theories with continuous time. On the other hand it is known $[1,3]$ that for high enough temperatures $\beta^{-1}$ there is a region $g_{1}^{2}(\beta)<g^{2}<g_{2}^{2}(\beta)$, where spatial Wilson loops still show area law behaviour, while infinitely heavy quarks are deconfined.

Here I show the existence of a third phase with deconfinement for external quarks and perimeter law behaviour for spatial Wilson loops for discrete abelian groups $G$. In this phase the deconfinement is due to the discreteness of $G$, and not to high temperatures. Indeed we will be able to prove a lower bound $\tilde{g}^{2}(\beta)$ on the transition coupling $g_{c}^{2}$, which stays bounded away from zero in the limit $\beta \rightarrow \infty$.

In order to prove these results we are going to derive a "quantum contour" expansion similar to the expansion introduced in [10]. It is convenient to normalise the Hamiltonian $H_{\Omega}=H_{\Omega}^{(0)}+V_{\Omega}$ a little bit differently by setting

$$
\begin{gathered}
H_{\Omega}^{(0)}=\frac{g^{2}}{2} \sum_{\langle x y\rangle} \tilde{J}_{x y}, \\
\left(\tilde{\triangle}_{x y} \Phi_{x y}\right)\left(g_{x y}\right)=-\sum_{h_{x y}: d\left(h_{x y}, g_{x y}\right)=d_{0}} d_{0}^{-2} \Psi_{x y}\left(h_{x y}\right) .
\end{gathered}
$$

I again start with an expansion for $Z_{\hat{N}, \Omega}^{\lambda}=\operatorname{Tr}_{\mathscr{H}_{\Omega}} T^{N}$, where $T$ is defined as in Sect. 2 [clearly $\Delta_{x y}$ in (2.4) has to be replaced by $\widetilde{\Delta}_{x y}$ ]. But now we expand $T$ around

$$
S^{(0)} \equiv e^{W}
$$

instead of $T^{(0)}=e^{D}$. One obtains

$$
\begin{gathered}
T=\sum_{L \subset \Omega_{1}} S(L), \\
S(L)=\left[\prod_{l \in L}\left(\sum_{n_{l}=1}^{\infty} \frac{1}{n_{l} !} \int_{0}^{1} d t_{l}^{1} \ldots d t_{l}^{n_{l}}\right)\right] S(\mathbf{t}),
\end{gathered}
$$

where $S(\mathbf{t})$ is obtained from $S^{(0)}$ by inserting $D_{l}$ at the times $t_{l}^{1}, \ldots, t_{l}^{n l}$.

Next I introduce a base of $\mathscr{H}_{\Omega}=P_{0} \widetilde{H}_{\Omega}$ in which $S^{(0)}$ is diagonal. It is conveniant to write the group $G$ additively. For functions $g: \Omega_{1} \rightarrow G, f: \Omega_{2} \rightarrow G$, I denote by $d g$ and $d f$ the corresponding exterior derivative

$$
\begin{aligned}
& (d g)(p)=\sum_{l \in \partial p} g(l), \\
& (d f)(c)=\sum_{p \in \hat{\partial} \mathcal{C}} f(p) .
\end{aligned}
$$


For $g: \Omega_{1} \rightarrow G: l \mapsto g_{l}$

$$
\Psi_{g}\left(\mathbf{g}^{\prime}\right)=\prod_{l \in \Omega_{1}} \delta\left(g_{l}, g_{l}^{\prime}\right)
$$

Let $f: \Omega_{2} \rightarrow G$ be a function with $d f=0$; I call such a function a contour in $\Omega$ and denote the set of contours in $\Omega$ by $\mathscr{C}$. Since $\left(\Omega, \Omega_{1}, \Omega_{2}, \ldots, \Omega_{d}\right)$ is a closed subcomplex of $Z^{d}$ (we have chosen free boundary conditions) every function $f \in \mathscr{C}$ can be written as $f=d g$ with $g: \Omega_{1} \rightarrow G(d \geqq 2)$. I set

$$
\tilde{\Psi}_{f}=P_{0} \Psi_{g}
$$

whenever $f \in \mathscr{C}$ and $f=d g$. Note that $\widetilde{\Psi}_{f}$ does not depend on the choice of $g$ because $P_{0} \Psi_{g}=P_{0} \Psi_{g^{\prime}}$, whenever $d g=d g^{\prime}$. It is easy to show that for contours $f, f^{\prime}$ in $\Omega\left(\widetilde{\Psi}^{f}, \widetilde{\Psi}^{f^{\prime}}\right)=\left\|\widetilde{\Psi}^{f^{\prime}}\right\|^{2} \delta\left(f, f^{\prime}\right)$ with $\left\|\widetilde{\Psi}^{f}\right\|>0$. Hence

$$
\mathscr{B}=\left\{\Psi^{f}=\widetilde{\Psi}^{f} /\left\|\widetilde{\Psi}^{f}\right\|: f \in \mathscr{C}\right\}
$$

is a system of orthonormal vectors in $\mathscr{H}_{\Omega}$. Since the vectors $\Psi_{g}$ form a base of $\widetilde{\mathscr{H}}_{\Omega}, \mathscr{B}$ is an ONB (orthonormal base) for $\mathscr{H}_{\Omega}$. I call $\mathscr{B}$ the contour base of $\mathscr{H}_{\Omega}$. $W$ (and hence $S^{(0)}$ ) are diagonal in the base $\mathscr{B}$,

$$
W \Psi^{f}=W(f) \Psi^{f}, \quad W(f) \equiv-\frac{\mu}{2} \sum_{p \in \Omega_{2}}(d(0, f(p)))^{2} .
$$

[Recall that we have written $G$ additively. Consequently we write $d(0, \cdot)$ instead of $d(\mathbb{1}, \cdot)$.] But $S(\mathbf{t})$ and $S(L)$ are not because $\tilde{\Delta}_{x y}$ induces transitions

$$
\tilde{\Delta}_{x y} P_{0} \Psi_{g}=P_{0} \tilde{\Delta}_{x y} \Psi_{g}=-\sum_{h_{x y}: d\left(h_{x y}, 0\right)=d_{0}} d_{0}^{-2} P_{0} \Psi_{g+h},
$$

where the sum goes over functions $h$ such that $d(h(l), 0)=d_{0}$ if $l=\langle x y\rangle$ and $h(l)=0$ if $l \neq\langle x y\rangle$. For $\Psi^{f} \in \mathscr{B}$ one obtains

$$
\tilde{\Delta}_{x y} \Psi^{f}=-\sum_{h_{x y} \in \mathscr{U}} d_{0}^{-2} \Psi^{f+d h},
$$

where $\mathscr{U} \subset G$ contains the nearest enighbour of 0 in $G$, i.e. the elements $h \in G$ with $d(h, 0)=d_{0}$. Using (4.4) and the fact that $\mathscr{B}$ is on ONB for $\mathscr{H}_{\Omega}$ we obtain

$$
Z_{N, \Omega}^{\lambda, \mu}=\sum_{\begin{array}{c}
f_{1}, \ldots, f_{N} \in \mathscr{C} \\
L_{1}, \ldots, L_{N} \subset \Omega_{1}
\end{array}} \prod_{t=1}^{N}\left(\Psi^{f_{t}}, S\left(L_{t}\right) \Psi^{f_{t+1}}\right),
$$

where $f_{N+1}$ is to be identified with $f_{1}$.

For $L \subset \Omega_{1}$ I set $P(L):=\left\{p \in \Omega_{2}: \exists l \in L\right.$ with $\left.l \in \partial p\right\}$ and $P^{c}(L):=\Omega_{2} \backslash P(L)$; for a link $l=\langle x y\rangle$ in $\Omega$, I define $p(t, l)$ as the plaquette $((t, x),(t, \lambda),(t+1, \lambda),(t+1, x))$ in $\Lambda=\{1, \ldots, N\} \times \Omega$. I call $K=\left(f_{1}, \ldots, f_{N}, L_{1}, \ldots, L_{N}\right)$ with $f_{i} \in \mathscr{C}, L_{i} \subset \Omega$ an allowed configuration, if $f_{t}=f_{t+1}$ on $P^{c}\left(L_{t}\right)(t=1, \ldots, N)$ and define the activity $z(K)$ as

$$
z(K)=\prod_{t=1}^{N}\left(\Psi^{f_{t}}, S\left(L_{t}\right) \Psi^{f_{t+1}}\right)
$$

Finally the support $\operatorname{supp}(K)$ of a configuration $K=\left(f_{1}, \ldots, f_{N} L_{1}, \ldots, L_{N}\right)$ is defined as the set of vertical and horizontal plaquettes $p(t, l)$ and $(t, p)$ such that $l \in L_{t}$ and $p \in \operatorname{supp} f_{t}$, respectively. Note that only allowed configurations contribute to (4.11) due to (4.10) and the fact that $S^{(0)}$ is diagonal in the basis $\mathscr{B}$. 
We now analyse the factorisation properties of $z(K)$. For $f \in \mathscr{C}$ and $L \subset \Omega_{1}$ let $\operatorname{supp}_{L} f=P(L) \cup \operatorname{supp} f$. We consider contours $f_{1}, f_{2}, g_{1}, g_{2}$ and sets $L_{1}, L_{2} \subset \Omega$, such that $f_{i}=g_{i}$ on $P^{c}\left(L_{i}\right)$. We say that $\left(f_{1}, L_{1}, g_{1}\right)$ and $\left(f_{2}, L_{2}, g_{2}\right)$ are compatible if there is no cube $c \in \Omega_{3}$ which contains a plaquette $p_{1} \in \operatorname{supp}_{L_{1}} f_{1}$ and a plaquette $p_{2} \in \operatorname{supp}_{L_{2}} f_{2}{ }^{6}$. Assume that $\left(f_{1}, L_{1}, g_{1}\right)$ and $\left(f_{2}, L_{2}, g_{2}\right)$ are compatible and set $L=L_{1} \cup L_{2}, f=f_{1}+f_{2}, g=g_{1}+g_{2}$. Then

$$
\left(\Psi^{f}, S(L) \Psi^{g}\right)=\prod_{i=1}^{2}\left(\Psi^{f i}, S\left(L_{i}\right) \Psi^{g i}\right) .
$$

This motivates the following definitions: We say two plaquettes $p, p^{\prime} \in \Omega_{2}$ are incompatible (or connected) in $\Omega$, if there is a cube $c$ in $\Omega$ such that $p \in \partial c$ and $p^{\prime} \in \partial c$. Two horizontal plaquettes $(t, p),\left(t^{\prime}, p^{\prime}\right)$ in $\Lambda$ are connected if $t=t^{\prime}$ and $p$ and $p^{\prime}$ are connected in $\Omega$. Two vertical plaquettes $p(t, l)$ and $p\left(t^{\prime}, l^{\prime}\right)$ in $\Lambda$ are called connected if $P(\{l\}) \cup P\left(\left\{l^{\prime}\right\}\right)$ is a connected set and $\left|t-t^{\prime}\right| \leqq 1$. A vertical plaquette $p(t, l)$ and a horizontal plaquette $\left(t^{\prime}, p^{\prime}\right)$ are called connected if $P(\{l\}) \cup p^{\prime}$ is a connected set and $t^{\prime}=t$ or $t^{\prime}=t+1$. An allowed configuration $K$ is called simple or a polymer if its support is a non-empty, connected set. Two polymers $K$ and $K^{\prime}$ are called compatible if $\operatorname{supp}(K) \cup \operatorname{supp}\left(K^{\prime}\right)$ is not a connected set. If

$$
K=\left(f_{1}, \ldots, f_{N}, L_{1}, \ldots, L_{N}\right)
$$

and

$$
K^{\prime}=\left(f_{1}^{\prime}, \ldots, f_{N}^{\prime}, L_{1}^{\prime}, \ldots, L_{N}^{\prime}\right)
$$

are compatible, $K+K^{\prime}$ denotes the configuration

$$
\left(f_{1}+f_{1}^{\prime}, \ldots, f_{N}+f_{N}^{\prime}, L_{1}, \cup L_{1}^{\prime}, \ldots, L_{N} \cup L_{N}^{\prime}\right) .
$$

Note that

$$
z(K)=\prod_{i=1}^{n} z\left(K_{i}\right)
$$

if $K_{1}, \ldots, K_{n}$ are pairwise compatible and $K=K_{1}+\ldots+K_{n}$.

Due to (4.11)-(4.13) $Z_{N, \Omega}^{\lambda, \mu}$ can be written as the partition function of the polymer system just defined

$$
Z_{N, \Omega}^{\lambda, \mu}=\sum_{n=0}^{\infty} \sum_{\left\langle K_{1}, \ldots, K_{n}\right\rangle} \phi\left(K_{1}, \ldots, K_{n}\right) \prod_{i=1}^{n} z\left(K_{i}\right)
$$

(as usual, $\phi$ takes values 1 or 0 . depending on whether $K_{1}, \ldots, K_{n}$ are pairwise compatible or not). For $\log Z_{N, \Omega}^{\lambda, \mu}$ one obtains the Mayer expansion

$$
\log Z_{N, \Omega}^{\lambda \cdot \mu}=\sum_{n=1}^{\infty} \sum_{K_{1}, \ldots, K_{n}} \frac{\Phi_{c}\left(K_{1}, \ldots, K_{n}\right)}{n !} \prod_{i=1}^{n} z\left(K_{i}\right) .
$$

The following lemma gives absolute convergence of the expansion (4.15) provided $|\lambda|$ is small enough and $\operatorname{Re} \mu$ is large enough.

\footnotetext{
${ }^{6}$ Note that $\operatorname{supp}_{L_{1}} f_{i}=\operatorname{supp}_{L_{1}} g_{i}$ because $f_{i}=g_{i}$ on $P^{c}\left(L_{i}\right)$
} 
Lemma 4.1. Let $G$ be a finite abelian group, $\mathscr{U}=\left\{g \in G: d(g, 0)=d_{0}\right\}$, and $\omega_{3}, \omega_{4}$, $e^{-b}$ the constants

$$
\begin{gathered}
\omega_{3}=\sum_{g \neq 0} e^{-\operatorname{Re}(\mu / 2) d^{2}(g, 0)}, \\
\omega_{4}=e^{\tilde{\omega}}-1, \quad \tilde{\omega}=|\mathscr{U}| d_{0}^{-2}|\lambda|, \\
e^{-b}=\max \left\{\omega_{3}, \omega_{4}^{1 / 2},\left(\omega_{3}+1\right) \omega_{4}^{1 / 4(d-1)}\right\} .
\end{gathered}
$$

Assume that $e^{-b} \leqq 1$. Then $z(K)$ is jointly analytic in $\mu$ and $\lambda$ and

$$
\sum_{K: \operatorname{supp}(K)=\mathscr{K}}|z(K)| \leqq e^{-b|\mathscr{K}|}
$$

for all connected sets $\mathscr{K}$ of plaquettes in $\Lambda$.

Proof. Let $L_{t}=\left\{l \in \Omega_{1}: p(t, l) \in \mathscr{K}\right\}$ and $P_{t}=\left(p \in \Omega_{2}:(t, p) \in \mathscr{K}\right\}$. Then

$$
\sum_{K: \operatorname{supp}(K)=\mathscr{K}}|z(K)|=\sum_{\substack{f_{1}, \ldots, f_{N} \in \mathscr{C} \\ \operatorname{supp} f_{i}=P_{\imath}}} \prod_{i=1}^{N}\left|\left(\Psi^{f i}, S\left(L_{i}\right) \Psi^{f_{i+1}}\right)\right| .
$$

I first bound sums of the form

$$
\sum_{\substack{f_{1} \ldots, f_{N} \in \mathscr{C} \\ \operatorname{supp} f_{i}=P_{i}}} \prod_{i=1}^{N}\left|\left(\Psi^{f i}, S\left(\mathbf{t}_{i}\right) \Psi^{f_{i+1}}\right)\right|,
$$

where $\mathbf{t}_{i}$ is a function which assigns to each $l \in L_{i}$ exactly $n(i, l)$ times $t_{i, l}^{1}, \ldots, t_{i, l}^{n(i, l)}$ between 0 and 1. Using (4.5), the bounds on (4.18) will give the desired bounds on (4.17). I take a typical term of the form (4.18), namely

$$
\mathscr{T}=\sum_{\substack{f_{1}, \ldots, f_{N} \in \mathscr{C} \\ \text { supp } f_{l}=P_{i}}} \prod_{i=1}^{N}\left|\left(\Psi^{f_{i}}, e^{s_{i} W} D_{l_{l}} e^{\tilde{s}_{i} W} D_{l_{l}^{\prime}} e^{s_{i} W} \Psi^{f_{i}+1}\right)\right|,
$$

with $0 \leqq s_{i}, \tilde{s}_{i}, s_{i}^{\prime} \leqq 1, s_{i}+\tilde{s}_{i}+s_{i}^{\prime}=1$. Inserting the base $\mathscr{B}$ we obtain

$$
\mathscr{T} \leqq \sum_{\substack{f_{l}, g_{l} \in \mathscr{C} \\ \operatorname{supp} f_{i}=P_{i}}} \prod_{i=1}^{N}\left\{e^{s_{l} \tilde{W}\left(f_{l}\right)} \widetilde{D}_{l_{l}}\left(f_{i}, g_{i}\right) \times e^{\tilde{S}_{i} \tilde{W}\left(g_{l}\right)} \widetilde{D}_{l_{l}^{\prime}}\left(g_{i}, f_{i+1}\right) e^{S_{l^{\prime}} \tilde{W}\left(f_{l}+1\right)}\right\}
$$

where

$$
\tilde{W}(f)=-\operatorname{Re}\left(\frac{\mu}{2}\right) \sum_{p \in \Omega_{2}} d(0, f(p))^{2}, \quad \tilde{D}_{l}(f, g)=\left|\left(\Psi^{f}, D_{l} \Psi^{g}\right)\right| .
$$

Next we remark that due to the "transition rules" (4.10) only contours $g_{i}$ with $\widetilde{P}_{i} \subset \operatorname{supp}\left(g_{i}\right) \subset \bar{P}_{i}$ contribute to the above sum. Here $\bar{P}_{i}=P_{i} \cup P\left(L_{i}\right)$ and $\widetilde{P}_{i}$ $=P_{i} \backslash P\left(L_{i}\right)$. I define

$$
\chi_{\tilde{P}, \tilde{P}}(g)=\left\{\begin{array}{ll}
1 & \text { if } \tilde{P} \subset \operatorname{supp}(g) \subset \bar{P} \\
0 & \text { otherwise }
\end{array} .\right.
$$

Then

$$
\begin{aligned}
& \mathscr{T} \leqq \sum_{f_{l}, g_{i} \in \mathscr{C}} \prod_{i=1}^{N}\left\{\chi_{\tilde{P}_{i}, \bar{P}_{i}}\left(f_{i}\right) e^{S_{i} \tilde{W}\left(f_{i}\right)} \tilde{D}_{l_{l}}\left(f_{i}, g_{i}\right)\right. \\
& \left.\times \chi_{\tilde{P}_{l}, \bar{P}_{l}}\left(g_{i}\right) e^{\tilde{s}_{\imath} \tilde{W}\left(g_{l}\right)} \widetilde{D}_{l_{i}}\left(g_{i}, f_{i+1}\right) \chi_{\tilde{P}_{i+1}, \bar{P}_{i+1}}\left(f_{i+1}\right) e^{s_{i}^{i} \tilde{W}\left(f_{i}+1\right)}\right\} .
\end{aligned}
$$


The right-hand side can be viewed as trace in $l^{2}(\mathscr{C})$. Using Hölder's inequality we obtain the bound

$$
\mathscr{T} \leqq \prod_{i=1}^{N} \sum_{\substack{f_{i} \in \mathscr{C} \\ \tilde{P}_{i} \subset \operatorname{supp} f_{i} \subset \bar{P}_{i}}} \tilde{\kappa}^{2} e^{\tilde{W}\left(f_{i}\right)},
$$

where $\tilde{\kappa}$ is the norm of the operator $\tilde{D}_{l}$ in $L^{2}(\mathscr{C})$ with kernel $\widetilde{D}_{l}(\cdot, \cdot)$. Bounding $\tilde{\kappa}$ by $\tilde{\omega}$ we obtain

$$
\mathscr{T} \leqq \prod_{i=1}^{N}\left\{\omega_{3}^{\left|\tilde{P}_{i}\right|}\left(1+\omega_{3}\right)^{\left|\vec{P}_{i} \backslash \tilde{P}_{i}\right|} \tilde{\omega}^{2}\right\}
$$

If one considers the general term in (4.18) instead of $\mathscr{T}, \tilde{\omega}^{2}$ has to be replaced by $\prod_{l \in L_{l}} \tilde{\omega}^{n(i, l)}$. Using (4.5) we finally get the bound

$$
\prod_{i=1}^{N}\left\{\omega_{3}^{\left|P_{i} \backslash P(L)\right|}\left(1+\omega_{3}\right)^{\left|P\left(L_{i}\right)\right|} \omega_{4}^{\left|L_{i}\right|}\right\}
$$

for the sum (4.17), where we used that $\widetilde{P}_{i} \backslash \widetilde{P}_{i}=P(L)$. Since every link $l \in \Omega_{1}$ is contained in at most $2(d-1)$ plaquettes $p \in \Omega_{2}$ we can bound

$$
\omega_{4}^{\left|L_{i}\right|}=\omega_{4}^{\frac{1}{2}\left|L_{i}\right|} \omega_{4}^{\frac{1}{2}\left|L_{i}\right|} \leqq \omega_{4}^{\frac{1}{2}\left|L_{i}\right|} \omega_{4}^{\frac{1}{4(d-1)}}\left|P\left(L_{i}\right)\right|
$$

provided $\omega_{4} \leqq 1$. Using the fact that

$$
|\mathscr{K}|=\sum_{i=1}^{N}\left(\left|L_{i}\right|+\left|P_{i}\right|\right) \leqq \sum_{i=1}^{N}\left(\left|L_{i}\right|+\left|P_{i} \backslash P\left(L_{i}\right)\right|+\left|P\left(L_{i}\right)\right|\right),
$$

we finally obtain the bound (4.16)

The following Corollary 4.2, as well as Theorem 4.3 need no explicit proof at this point.

Corollary 4.2. Let $G$ be a finite abelian group, $\chi$ the character defining the hamiltonian $H$, and $d \geqq 2$ the space dimension. Then there are constants $g_{1}^{2}>0$ and $\beta_{1}<\infty$, depending only on $G, \chi$ and $d$, such that the following statement is true: $\forall \beta>0 \forall g^{2}$ with

$$
\operatorname{Re} \frac{1}{g^{2}}>\frac{1}{\tilde{g}^{2}(\beta)} \equiv \frac{1}{g_{1}^{2}} \max \left\{1, \beta_{1} / \beta\right\}
$$

$\exists N, \mu, \lambda$ such that i) $Z_{\beta, \Omega}=Z_{N, \Omega}^{\lambda, \mu}$ and ii) the Mayer expansion (4.15) for $\log Z_{N . \Omega}^{\lambda, \mu}$ is absolutely convergent. $\log Z_{\beta, \Omega}$ is an analytic function of $\mathrm{g}^{2}$ in the region

$$
\mathscr{G}_{1}(\beta)=\left\{g^{2} \in \mathbb{C} \backslash\{0\}: \operatorname{Re} \frac{1}{g^{2}}>\frac{1}{\tilde{g}^{2}(\beta)}\right\} .
$$

Theorem 4.3. i) Let $d \geqq 2, \beta>0$ and $\operatorname{Re}\left(1 / g^{2}\right)>1 / \tilde{g}^{2}(\beta)$. Then the infinite volume limit

$$
\langle A\rangle_{\beta}-\lim _{\Omega \rightarrow Z^{d}}\langle A\rangle_{\beta, \Omega}
$$

exists for all local observables $A \in \mathscr{A} .\langle\cdot\rangle_{\beta}$ is translation invariant and $\langle A\rangle_{\beta}$ is an analytic function of $g^{2}$ in the region $\mathscr{G}_{1}(\beta)$. 
ii) For $\beta>0$ and $\operatorname{Re}\left(1 / g^{2}\right)>1 / \tilde{g}^{2}(\beta)$ and $\forall A, B \in \mathscr{A}$ there are constants $K_{A B}<\infty$ and $M>0$ such that

$$
\left|\left\langle A t_{x}(B)\right\rangle_{\beta}-\langle A\rangle_{\beta}\langle B\rangle_{\beta}\right| \leqq K_{A B} e^{-M|x|}
$$

$\forall x \in \Omega . M>0$ can be chosen independently of $A$ and $B$.

iii) $\forall g^{2}$ with $\operatorname{Re}\left(1 / g^{2}\right)>1 / g_{1}^{2}$ the "full thermodynamic limit (1.7) exists. $\langle\cdot\rangle$ is translation invariant and $\langle A\rangle$ can be continued to an analytic function of $g^{4}$ in the region

$$
\mathscr{G}_{1}=\left\{g^{4} \in \mathbb{C}:\left|g^{4}\right|<g_{1}^{4}\right\} .
$$

In addition the statements analogous to Theorem 3.4, ii) and Remark i), Sect. 3 are true, i.e. $\forall g^{2}>0$ with $g^{2}>g_{1}^{2}$ the infinite volume Hamiltonian $H$ exists and has a mass gap $\geqq M$.

Theorem 4.4. Let $d \geqq 3, \beta>0$ and $\operatorname{Re}\left(1 / g^{2}\right)>1 / \tilde{g}^{2}(\beta)$. Then horizontal Wilson loops show perimeter law behaviour, i.e.

$$
\left|\left\langle\chi_{q}\left(g_{C}\right)\right\rangle_{\beta}\right| \geqq e^{-\gamma|C|}
$$

with a constant $\gamma<\infty$, which does not depend on $C$.

Remark $i$. The $1+1$ dimensional theory on a symmetric space-time lattice shows area law behaviour for all couplings $g^{2}>0$. Using a correlation inequality (see [3]) one concludes that $\left\langle\chi_{q}\left(g_{C}\right)\right\rangle_{\beta}$ shows area law behaviour for all $g^{2}>0$ and $\beta<\infty$ in $2+1$ dimensions.

Proof of Theorem 4.4. I consider the modified partition function

$$
Z_{N, \Omega}^{\lambda, \mu}(C)=\operatorname{Tr}_{\mathscr{H}_{\Omega}} \chi_{q}\left(g_{C}\right) T^{N}
$$

Since $\chi_{q}\left(g_{C}\right)$ is diagonal in the contour base $\mathscr{B}$ and

$$
\left(\Psi^{f}, \chi_{q}\left(g_{C}\right) \Psi^{f}\right)=\prod_{i=1}^{N}\left(\Psi^{f i}, \chi_{q}\left(g_{C}\right) \Psi^{f i}\right)
$$

$\forall f \in \mathscr{C}$ with $f=f_{1}+\ldots+f_{n}$ and $f_{i} \in \mathscr{C}, Z_{N, \Omega}^{\lambda, \mu}(C)$ can be written as a partition function with modified activities

$$
Z_{N, \Omega}^{\lambda, \mu}(C)=\sum_{n=0}^{\infty} \sum_{\left\{K_{1}, \ldots, K_{n}\right\}} \Phi\left(K_{1}, \ldots, K_{n}\right) \prod_{i=1}^{n} z_{C}\left(K_{t}\right)
$$

where for a configuration $K=\left(f_{1}, \ldots, f_{N}, L_{1}, \ldots, N_{N}\right)$,

$$
z_{C}(K)=z(K) \prod_{i=1}^{N}\left(\Psi^{f i}, \chi_{q}\left(g_{C}\right) \Psi^{f i}\right) .
$$

The Mayer expansion for $\log Z_{N, \Omega}^{\lambda, \mu}(C)$, combined with that for $\log Z_{N, \Omega}^{\lambda, \mu}$, gives

$$
\log \left\langle\chi_{q}\left(g_{C}\right)\right\rangle_{N, \Omega}^{\lambda, \mu}=\sum_{n=1}^{\infty} \sum_{K_{1}, \ldots, K_{n}} \frac{\Phi_{c}\left(K_{1}, \ldots, K_{n}\right)}{n !}\left(\prod_{i=1}^{n} z_{C}\left(K_{i}\right)-\prod_{i=1}^{n} z\left(K_{i}\right)\right) .
$$

Telescoping the difference of the products and using the bound $\left|z_{C}(K)\right| \leqq|z(K)|$ we obtain the bound

$$
\left|\log \left\langle\chi_{q}\left(g_{C}\right)\right\rangle\right| \leqq \sum_{n=1}^{\infty} \sum_{K_{1}, \ldots, K_{n}}\left|\frac{\Phi_{c}\left(K_{1}, \ldots, K_{n}\right)}{n !}\right| \times n\left|z_{C}\left(K_{1}\right)-z\left(K_{1}\right)\right| \prod_{i=2}^{n}\left|z\left(K_{i}\right)\right|
$$


We now proceed as in the proof of the perimeter law for the Wilson-type theory (see e.g. [7]). Note that there are not more than $|C|$ const $^{s}$ contours $f$ with connected support of size $s$ in three and more space dimensions, for which $\left(\Psi^{f}, \chi_{q}\left(g_{C}\right) \Psi^{f}\right) \neq 1$, because this implies that $f$ winds around $C$.

Remarks. ii) In Theorems 4.3 and 4.4 we assume that $G$ is a finite abelian group. As can be seen from the proof of Lemma 4.1, the finiteness of $G$ is not essential, it is enough to assume that $G$ is discrete because only the finiteness of $\mathscr{U}$ (see Lemma 4.1) was used.

iii) The "quantum contour" expansion derived in this section has given several rigorous results concerning the expectation values of local observables $A \in \mathscr{A}$ (including horizontal Wilson loops). It is not obvious, however, how this expansion can be used to prove deconfinement in the sense of Polyakov. This does not mean, however, that the theory does not show deconfinement in the region $\mathscr{G}_{1}(\beta)$. In fact deconfinement in the sense of Polyakov can be shown by other methods, at least in a certain subregion of $\mathscr{G}_{1}(\beta)$, provided $d \geqq 2$ (see Remark iv).

iv) The deconfinement proof of Borgs and Seiler [1] works for discrete groups as well as for continuous groups, but only for $d \geqq 3$. To prove deconfinement for finite abelian groups $\forall d \geqq 2$, one might proceed for example as follows:

By a correlation inequality the 2-point function $G_{q \bar{q}}^{\beta}=e^{-\beta F_{q \bar{q}}^{\beta}}$ is bounded from below by the 2-point function $\widetilde{G}_{q \bar{q}}^{\beta}$ without potential term. $\widetilde{G}_{q \bar{q}}^{\beta}$, however, can be written as the 2-point function in a $G$-valued spin system at temperature $\beta g^{2}$. Long range order for this spin system for $g^{2} \beta<O(1)$ implies deconfinement for the gauge theory in $d \geqq 2$, provided $0<g^{2}<$ const $\beta^{-1}$.

v) It is clear that the "quantum contour expansion" described in this section has to be modified for non-abelian groups, because for non-abelian groups equivalence classes of gauge equivalent classical configurations cannot be described by the corresponding plaquette variables ${ }^{7}$. The details will be discussed in Appendix B.

\section{Discussion}

In this paper we proved several rigorous results concerning the phase structure of lattice Yang-Mills theories with continuous time: For arbitrary compact gauge groups $G$ we derived a convergent strong coupling expansion proving confinement in the sense of Polyakov, exponential clustering for local observables and an area law for spatial Wilson loops in a region

$$
\operatorname{Reg}^{2}>\bar{g}^{2}(\beta)=g_{0}^{2} \max \left\{1, \beta_{0} / \beta\right\} .
$$

This result should be compared to that of [1], where deconfinement was shown for

$$
g^{2}<g_{2}^{2}(\beta)=\operatorname{const} \beta^{-1},
$$

provided $d \geqq 3$. Since spatial Wilson loops do not provide a confinement criterion for the theory at nonzero temperature, this does not exclude an area law for spatial

\footnotetext{
${ }^{7}$ This fact was overlooked in [7], where the weak coupling expansion for the Wilson theory was treated on equal footings for finite abelian and finite non-abelian groups
} 
Wilson loops for $g^{2}<g_{2}^{2}(\beta)$. In fact there are convincing arguments $[3,8]$ for the conjecture that spatial Wilson loops show area law behaviour for all $\beta<\infty$ and $g^{2}>0$, provided $\left.G=U(1)\right)$ or $S U(N)$ and $d+1 \leqq 4$ or $d+1 \leqq 5$, respectively. For $G=U(1)$ this conjecture has been proven in [3]; for arbitrary compact groups (discrete or continuous) this area law behaviour has been proven for

$$
g^{2}>g_{1}^{2}(\beta)=\operatorname{const}^{\prime} \beta \text {. }
$$

The above discussion is summarised in Fig. 2; I is the deconfinement region (5.2) established in [1], II the confinement region (5.1) established in Sects. 2 and 3 of this paper. In addition I indicates the generally assumed phase transition line $\Gamma$ $[G=U(1)$ or $S U(N), d+1=4]$. Area law behaviour for spatial Wilson loops has been proven for all $g^{2}>0, \beta<\infty$ for $G=U(1)$; for $G=S U(N)$ the same should be true, but rigorously it is only known in the region (5.3). The corresponding bound is indicated by a dashed line. Exponential clustering has been shown in the confinement region II; for $G=S U(N)$ it should, however, also be true in the deconfinement region, at least for $N=2,3$ (see [8]). For large $N$ the deconfinement region might decompose into a Coulomb-phase and a phase with exponential clustering, see [1]. For $G=U(1)$ one does not expect exponential clustering in the deconfinement phase.

The phase diagram for discrete compact groups (we treated the abelian case in Sect 4; the non-abelian case is discussed in Appendix B) is quite different (Fig. 3). Here the deconfinement region decomposes into (at least) two phases which can be
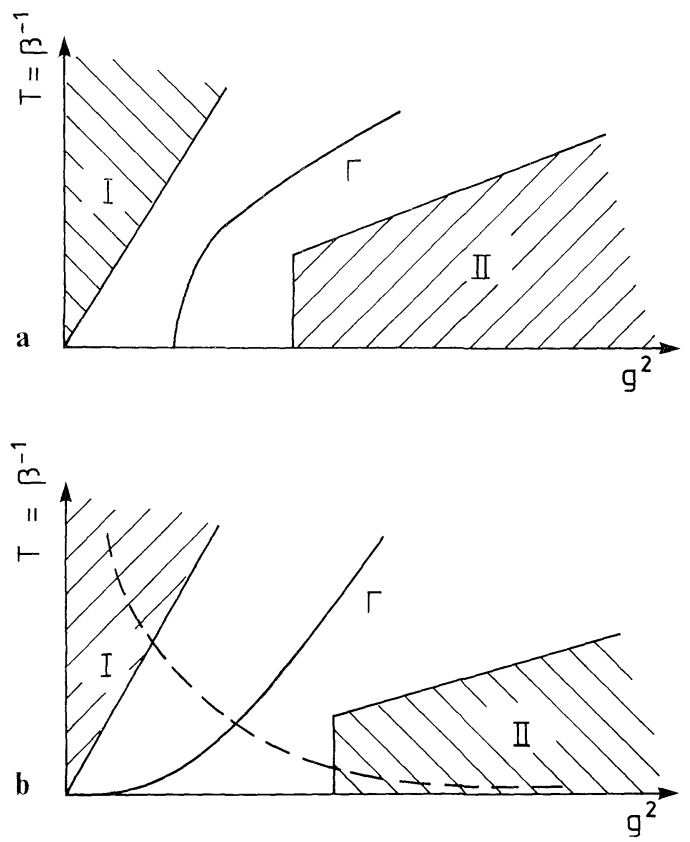

Fig. 2. a The phase diagram for $G=U(1)$ in $d+1=4$ dimensions. b The phase diagram for $G=S U(N)$ in $d+1=4$ dimensions 


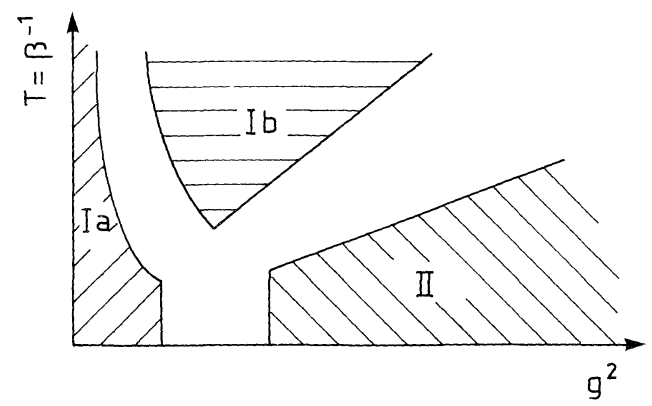

Fig. 3. The phase diagram for discrete compact groups in $d+1 \geqq 4$ dimensions

distinguished by the behaviour of spatial Wilson loops. In the region $\mathrm{I} b{ }^{8}$ where deconfinement is due to high temperatures, they still show area law behaviour, whereas in the region Ia, where deconfinement is due to the discreteness of the gauge group, they show perimeter law behaviour (provided $d+1 \geqq 4$ ). ${ }^{9}$ Deconfinement in the sense of Polyakov was proven in the subregion

$$
\left\{g^{2}<\operatorname{const} \beta^{-1} ; \operatorname{Re}\left(1 / g^{2}\right)>\tilde{g}^{2}(\beta)\right\}
$$

of region Ia, but it should be true in the whole region Ia.

Exponential clustering was shown in region Ia and in region II, but not in region $\mathrm{Ib}$. The following cluster expansion, however, is convergent in the region $\mathrm{Ib}$ and implies exponential clustering in the region $\mathrm{Ib}$.

I first consider the theory without potential term. This theory corresponds to a $G$-valued classical spin system in $d$ dimensions and can be handled by a standard Peierls contour expansion, provided the temperature $\beta g^{2}$ of this spin system is small enough. To include the potential term one combines this expansion with a Duhamel or Trotter product expansion in $V$. The resulting "high/lowtemperature" expansion is convergent provided $\beta g^{2}$ and $\beta / g^{2}$ are small.

I close this section with some remarks concerning the analyticity properties of the string tension $\sigma_{q}$ at zero temperature. I again consider the rescaled theory with Hamiltonian

$$
H_{\Omega}^{\prime}=\frac{1}{g^{2}} H_{\Omega}=\frac{1}{2} \sum_{\langle x y\rangle} A_{x y}-\frac{1}{2 g^{4}} \sum_{p} d\left(\mathbb{1}, g_{\hat{o} p}\right)^{2} .
$$

The string tension $\sigma_{q}^{\prime}$ in the rescaled theory and that one in the original theory are related by

$$
\sigma_{q}=g^{2} \sigma_{q}^{\prime}
$$

The following Quasi-theorem can be proven by a combination of the methods developed in [18, Chap. 2] (see [22] for a short review) and those of Sects. 2 and 3

\footnotetext{
${ }^{8}$ Region Ia corresponds to the inequality

$$
\operatorname{Re}\left(1 / g^{2}\right)>1 / \tilde{g}^{2}(\beta)
$$
}

with $\tilde{g}^{2}(\beta)$ according to Corollary 4.2 , region $\mathrm{Ib}$ is the region where both (5.2) and (5.3) are true, and region II is characterised by the inequality (5.1)

${ }^{9}$ The theory in $2+1$ dimensions probably shows an area law behaviour for all $g^{2}>0$ and all $\beta<\infty$; for abelian groups this was shown in [3]; see also Remark i), Sect. 4 
of the present article. To simplify our notation we stick to the groups $G=U(N)$ or $G=S U(N)$.

Quasi-Theorem 5.1. Let $v=d+1 \geqq 3$ and assume that $q$ is the fundamental representation of $G=U(N)$ or $S U(N)$. Then $\sigma_{q}^{\prime}=\sigma_{q} / g^{2}$ is an analytic function of $1 / g^{4}$ in a neighbourhood of zero.

Sketch of Proof. Fix a constant $\lambda>0$ to be chosen later and consider the two point function

$$
G_{N}(l)=\lim _{\Omega \rightarrow Z^{d}} G_{N, \Omega}^{\lambda, \mu=2 \lambda / g^{4}}\left(0, l e_{1}\right)
$$

where $e_{1}$ is the unit lattice vector in 1-direction. Denote the Polyakov loops in $\Lambda=\{1, \ldots, N\} \times \Omega$ which pass through 0 and $\left(0, l_{1}\right)$ by $C_{0}$ and $C_{l}$, respectively. We consider the cluster expansion $(2.35)$ for $G_{N}(l)$. For large $g^{2}$ the leading polymer $\gamma_{0}$ will contain no cubes, because the activity of a polymer $\gamma$ falls like $g^{-4 k}$ with the number $k$ of cubes in $\gamma$. So $\gamma_{0}$ consists of the plaquettes in the minimal surfaces $S_{0}$ with boundary $C_{0} \cup C_{l}$. As in [18], Chap. 2, we consider the deviations from $\gamma_{0}$ as a gas of particles living on $\gamma_{0} . G_{N}(l)$ [more precisely $\left.G_{N}(l) / z\left(\gamma_{0}\right)\right]$ is its partition function and $\sigma^{\prime}$ its free energy density. For large $\lambda$ and small $\lambda / g^{4}$ this gas is dilute and the resulting Mayer expansion for $\sigma^{\prime}$ is convergent. Note that the excitations of $\gamma_{0}$ may contain cubes and plaquettes while they were only built out of plaquettes in the case considered in [18]. This adds some complications to the geometrie analysis of the surfaces bounded by $C_{0} \cup C_{1}$, but there is no doubt that the above theorem can be proven with the technique developed in [18].

Remarks. i) The above "proof" clearly gives also the analyticity of the quarkantiquark potential $V_{q \bar{q}}^{\beta=0}$ in a region $\left\{1 / g^{4}:\left|1 / g^{4}\right|<0(1)\right\}$, as well as certain properties of the asymptotic behaviour of $V_{q \bar{q}}(l)$, as $l \rightarrow \infty$; cf. [18, Theorem 2.2].

ii) Combining the above techniques with the particle analysis of Bricmont and Fröhlich [23], one obtains the analyticity of $M / g^{2}$, where $M$ is the mass gap, in a neighborhood of $1 / g^{4}=0$.

iii) One can use the techniques of the above "proof" to show that the perturbative expansion (in $1 / g^{2}$ ) for $\sigma_{q}$ and for $M$ (as sketched in [5]) is convergent for large $g^{2}$.

\section{Appendix A}

In this appendix I construct a translation invariant infinite volume vacuum $\left(\beta^{-1}=0\right)$ state $\langle\cdot\rangle$ for arbitrary couplings $g^{2}>0$. I start with a sequence $\Omega^{(n)}$ of finite boxes

$$
\Omega^{(n)}=\left\{x \in Z^{d}:\left|x_{i}\right| \leqq L_{n}\right\} \nearrow Z^{d}
$$

with periodic boundary conditions and the corresponding vacuum states on $\mathscr{A}_{\Omega^{(n)}}$

$$
\omega_{n}(A)=\lim _{\beta \rightarrow \infty}\langle A\rangle_{\beta, \Omega^{(n)}}
$$

(use the Perron-Frobenius theorem to show the existence of the limit $\beta \rightarrow \infty$ ). Using a lemma of Glimm and Jaffe [13] and a bound on $\omega_{n}\left(\Delta_{x y}\right)$ which is uniform in $n, \mathrm{I}$ 
show the existence of a weakly convergent subsequence $\omega_{n(i)}(\cdot)$. The limit

$$
\langle\cdot\rangle=\lim _{i \rightarrow \infty} \omega_{n(i)}(\cdot)
$$

is the desired translation invariant infinite volume vacuum.

Remarks. i) In fact we will show the stronger statement that $\omega_{n(i)}$ is locally norm convergent, i.e. for any bounded region $\Omega \subset Z^{d}$,

$$
\left.\left.\omega_{n(i)}\right|_{\mathscr{A}_{\Omega}} \rightarrow\langle\cdot\rangle\right|_{\mathscr{A} \Omega} \text { in norm } .
$$

ii) From i) it follows immediately that $\langle\cdot\rangle$ is locally normal, because a local norm limit of normal states is locally normal.

The following lemma states the desired uniform bound on $\omega_{n}\left(\Delta_{x y}\right)$.

Lemma A.1. Let $g^{2}>0$. Then

$$
0 \leqq \omega_{n}\left(\Delta_{x v}\right) \leqq \frac{(d-1) \chi(\mathbb{1})}{g^{4}}
$$

for all links $\langle x y\rangle$ in $\Omega^{(n)} ; \chi(\cdot)$ is the character used in the definition of the potential $V_{\Omega}$ (see Sect. 1).

Proof. Let $E(\Omega)=\inf \operatorname{spec} H_{\Omega}$. Then

$$
E\left(\Omega^{(n)}\right) \leqq\left\langle 1\left|H_{\Omega^{(n)}}\right| 1\right\rangle=\left\langle 1\left|V_{\Omega^{(n)}}\right| 1\right\rangle=\frac{\chi(\mathbb{1})}{g^{2}} \times\left|\Omega_{2}^{(n)}\right|=\frac{(d-1) \chi(\mathbb{1})}{2 g^{2}} \times\left|\Omega_{1}^{(n)}\right|,
$$

where $|1\rangle$ is the constant function 1 in $L^{2}\left(d g_{\Omega^{(n)}}\right)$ and $\Omega_{1}^{(n)}, \Omega_{2}^{(n)}$ denote the sets of links and plaquettes in $\Omega^{(n)}$. Using the translation invariance of $\omega_{n}$ and the stability of $V_{\Omega}$ (with our choice of normalisation, $V_{\Omega} \geqq 0$ ) we get for $\langle x y\rangle \in \Omega_{1}^{(n)}$

$$
\begin{aligned}
\omega_{n}\left(\Delta_{x y}\right) & =\frac{2}{g^{2}} \frac{1}{\left|\Omega_{1}^{(n)}\right|} \omega_{n}\left(H_{\Omega^{(n)}}^{(0)}\right)=\frac{2}{g^{2}} \frac{1}{\left|\Omega_{1}^{(n)}\right|}\left[\omega_{n}\left(H_{\Omega^{(n)}}\right)-\omega_{n}\left(V_{\Omega^{(n)}}\right)\right] \\
& \leqq \frac{2}{g^{2}} \frac{1}{\left|\Omega_{1}^{(n)}\right|} \omega_{n}\left(H_{\Omega^{(n)}}\right) \leqq \frac{2(d-1) \chi(\mathbb{1})}{g^{4}}
\end{aligned}
$$

where we have used (A.3) and the fact that $E\left(\Omega^{(n)}\right)=\omega_{n}\left(H_{\Omega^{(n)}}\right)$ in the last step.

To continue we need the following lemma, which is proven in [13].

Lemma A.2 (= Lemma 4.7 of [13]). Let $\mathscr{B}$ be the algebra of all bounded operators on some separable Hilbert space $\mathscr{H}$ and let $N>0$ be an operator on $\mathscr{B}$ with compact inverse $N^{-1} \in \mathscr{B}$. Then the set

$$
\{T \in \mathscr{B} \mid 0 \leqq T \leqq 1, \operatorname{Tr}(T N) \leqq k\}
$$

is compact in the trace norm for all $0<k<\infty$.

To apply the lemma we fix a finite volume $\Omega \subset Z^{d}$ and set $\mathscr{H}=L^{2}\left(d g_{\Omega}\right)$,

$$
\omega_{n, \Omega}=\left.\omega_{n}\right|_{\mathscr{A}_{\Omega}} \text {. }
$$

By construction $\omega_{n, \Omega}$ is normal, i.e. $\omega_{n, \Omega}(\cdot)=\operatorname{Tr} \cdot T_{n}$ with some operator $0 \leqq T_{n} \leqq 1$ on $\mathscr{H}$. We set

$$
N=\mathbb{1}+\sum_{\left\langle x y^{\rangle}\right\rangle \subset \Omega} \Delta_{x y} .
$$


Clearly $N^{-1}$ is compact and

$$
\omega_{n, \Omega}(N) \leqq 1+\frac{2(d-1) \chi(\mathbb{1})}{g^{4}}\left|\Omega_{1}\right|
$$

by Lemma A.1. We therefore can apply Lemma A.2 to obtain a subsequence $n(i)$ such that $\omega_{n(i), \Omega}$ is norm convergent on $\mathscr{A}_{\Omega}$. By the diagonal sequence trick $n(i)$ can be chosen independently of $\Omega$, which proves the convergence stated at the beginning of this appendix.

\section{Appendix B}

In this appendix I discuss the modifications which are needed to generalize the expansion of Sect. 4 to non-abelian groups. For simplicity of notation I assume $d=3$. The complex $\left(\Omega, \Omega_{1}, \Omega_{2}, \Omega_{3}\right)$ is now taken as the open subcomplex of $Z^{3}$ generated by $\Omega$ (this corresponds to Dirichlet boundary conditions). For later use I define $\partial \Omega$ as the set $\left\{x \in Z^{3} \backslash \Omega: \exists y \in \Omega\right.$ with $\left.\langle x y\rangle \in \Omega_{1}\right\}$.

As in Sect. 4 I set

$$
\Psi_{g}\left(\mathbf{g}^{\prime}\right)=\prod_{l \in \Omega_{1}} \delta\left(g_{l}^{-1} g_{l}^{\prime}\right)
$$

but a contour is now defined more abstractly as an equivalence class $[g]$ of gauge equivalent classical configurations. I set $\widetilde{\Psi}^{[g]}=P_{0} \Psi_{g}$ and claim that

$$
\mathscr{B}=\left\{\Psi^{[g]}: \Psi^{[g]}=K(\Omega)^{-1 / 2} \widetilde{\Psi}^{[g]}\right\}
$$

is an orthonormal basis of $\mathscr{H}_{\Omega}=P_{0} \tilde{\mathscr{H}}_{\Omega} ; K(\Omega)$ is the constant $p^{\left|\Omega_{1}\right|-|\Omega|}$, where $p$ is the cardinality of $G$. Since $\tilde{\mathscr{B}}=\left\{\Psi_{g} \mid g: \Omega_{1} \rightarrow G\right\}$ is a basis of $\tilde{\mathscr{H}}_{\Omega}$ and $\left(\Psi_{g}, P_{0} \Psi_{g^{\prime}}\right)=0$ if $g$ and $g^{\prime}$ are not gauge equivalent, it only remains to show that

$$
\left\|P_{0} \Psi_{g}\right\|^{2}=K(\Omega)
$$

for all $g: \Omega_{1} \rightarrow G$. By an explicit calculation

$$
\left\|P_{0} \Psi_{g}\right\|^{2}=\int \prod_{x \in \Omega} d h_{x} \prod_{\langle x y\rangle \in \Omega_{1}} \delta\left(h_{x} g_{x y} h_{x}^{-1} g_{x y}^{-1}\right) .
$$

We show that for abelian as well as for non-abelian groups only $h \equiv \mathbb{1}$ contributes to the right-hand side. Consider a link $\langle x y\rangle \in \Omega_{1}$ such that one of its endpoints, say $x$, lies in $\partial \Omega$. Due to our choice of boundary conditions $h_{x}=\mathbb{1}$ for such an $x$. Using the invariance of the $\delta$-function and the fact that $\mathbb{1}$ commutes with $g_{x y}$, we find that only $h_{y}=\mathbb{1}$ contributes to the integral. Iterating this argument one proves that only $h \equiv \mathbb{1}$ gives a nonzero contribution to the integral. This proves (B.1) (note that $\delta(\mathbb{1})=p$, if the $\delta$-function is normalized in such a way that $\left.\int_{G} d g \delta(g)=1\right)$.

Consider the simplified model without kinetic term. Its partition function is

$$
\tilde{Z}_{\Omega}=\operatorname{Tr}_{\mathscr{H} \Omega} e^{-\beta V_{\Omega}} .
$$

Since $\mathscr{B}$ is an orthonormal basis for $\mathscr{H}_{\Omega}$ we can rewrite $\tilde{Z}_{\Omega}$ as a sum over contours.

$$
\tilde{Z}_{\Omega}=\sum_{[g]} e^{-\beta V([g)]}
$$

with $V([g])=\left(1 / 2 g^{2}\right) \sum_{p \in \Omega_{2}} d\left(\mathbb{1}, g_{\partial p}\right)^{2}$. 
I need some notation: For a set $W \subset \Omega_{3}$ I define $\bar{W}$ as the subset $\bar{W}:=\bigcup_{c \in W} c$ of $R^{3} ; \bar{P}$ for $P \in \Omega_{2}$ is defined accordingly. A connected set $W \subset \Omega_{3}$ is called a simply connected thick surface if $\bar{W}$ is simply connected. We say " $P_{1} \subset \Omega_{2}$ and $P_{2} \subset \Omega_{2}$ are separated by the simply connected thick surface $\bar{W}$ " if $\bar{\Omega}_{3} \backslash \bar{W}$ has two connectivity components $K_{1}, K_{2}$ such that i) $\bar{P}_{1} \subset K_{1}$ and $\bar{P}_{2} \subset K_{2}$, and ii) $\operatorname{dist}\left(K_{1}, K_{2}\right) \geqq 1$.

We call a non-empty set $P$ of plaquettes simple if it cannot be decomposed into sets $P_{1}, P_{2}$ which can be separated by a simply connected thick surface. $P_{1}, \ldots, P_{n}$ $\subset \Omega_{2}$ are called the components of a set $P \subset \Omega_{2}$ if i) $P=P_{1} \cup \ldots \cup P_{n}$, ii) $P_{1}, \ldots, P_{n}$ are simple, and iii) $P_{i}$ and $P_{j}$ can be separated by a simply connected thick surface for all $i \neq j$. I define: The support of a contour $[g]$ is the set of plaquettes $p$ for which $g_{\partial p} \neq \mathbb{1}$; a polymer is a contour with simple support; two polymers $\alpha_{1}, \alpha_{2}$ with support $P_{1}, P_{2}$, respectively, are compatible if $P_{1}, P_{2}$ are the components of $P_{1} \cup P_{2}$. I say $\tilde{\alpha}$ is a component of a contour $\alpha$, if $\operatorname{supp}(\tilde{\alpha})$ is a component of $\operatorname{supp}(\alpha)$ and if one can find gauge fields $g, \tilde{g}$ such that i) $\alpha=[g \tilde{g}]$, ii) $\tilde{\alpha}=[\tilde{g}]$ and iii) $\operatorname{supp}(g) \cap \operatorname{supp}(\tilde{g})=\emptyset$ (as a set of links in $\Omega_{1}$ ).

I claim that each contour $[g]$ can be decomposed in a unique way into pairwise compatible components $\left[g_{1}\right], \ldots,\left[g_{n}\right]$. For simplicity I only consider the case that $P=\operatorname{supp}([g])$ has two components (I denote them by $P_{1}$ and $P_{2}$ ) and leave the general case as an exercise. Let $W$ be a simply connected thick surface that separates $P_{1}$ and $P_{2}$, and $K_{1}$ and $K_{2}$ the connectivity components of $\bar{\Omega}_{3} \backslash \bar{W}$ which contain $P_{1}$ and $P_{2}$, respectively. Since $W$ is simply connected, we can find a gauge transformation $g \rightarrow \tilde{g}$, such that $\tilde{g}=\mathbb{1}$ for all links in $W$. I define $\left(g_{i}\right)_{l}=\tilde{g}_{l}$ if $l$ lies in $K_{i}$ and $\left(g_{i}\right)_{l}=\mathbb{1}$ if $l$ lies not in $K_{i}$. Obviously $\left[g_{1}\right]$ and $\left[g_{2}\right]$ are pairwise compatible components of $[g]$. To show uniqueness one has to show that the equivalence classes $\left[g_{1}\right]$ and $\left[g_{2}\right]$ do not depend on the particular choice of $W$ and $\tilde{g}$. This is an easy exercise.

Given the above decomposition of contours into pairwise compatible components one immediately obtains a representation of $\widetilde{Z}_{\Omega}$ as the partition function of a polymeter system. Note, however, that there is an additional combinatoric difficulty with respect to Sect. 4: To show diluteness of the polymer system one has to show that the number $n(s, \alpha)$ of polymers $\beta$ of size $s$ which are incompatible with a given polymer $\alpha$ is bounded by $|\alpha|$ const $^{s}$, where $|\alpha|$ denotes the number of plaquettes in the support of $\alpha$. This was easy in the abelian case, because there $\operatorname{supp}(\alpha)$ and $\operatorname{supp}(\beta)$ were connected sets. Here $\operatorname{supp}(\alpha)$ and $\operatorname{supp}(\beta)$ are not necessarily connected sets, but may be built out of several closed loops which wind around each other. With a little bit of experience with cluster expansions, however, it is not hard to show that a bound of the above form is nevertheless true.

The generalisation from the simplified model with Hamiltonian $H_{\Omega}=V_{\Omega}$ to the full model with Hamiltonian $H_{\Omega}=V_{\Omega}+H_{\Omega}^{(0)}$ is straightforward (use the Duhamel expansion to expand in $\left.H_{\Omega}^{(0)}\right)$ : as in Sect. 4 the kinetic term $H_{\Omega}^{(0)}$ induces transitions between different contours and we obtain a polymer system in $\Lambda=\{1, \ldots, N\} \times \Omega$. Up to technical details we thus have proven the following

Quasi-Theorem B.1. Let $d \geqq 3$. Then Theorems 4.3 and 4.4 remain valid for nonabelian discrete groups as well.

Acknowledgements. I thank Erhard Seiler, Simon Shlosman and Tom Kennedy for several discussions and helpful suggestions. I am also grateful to Krysztof Gawedzki and the IHES for hospitality during several periods where this work was done. 


\section{References}

1. Borgs, C., Seiler, E.: Lattice Yang-Mills theories at nonzero temperature and the confinement problem. Commun. Math. Phys. 91, 329 (1983)

2. Tomboulis, E.T., Yaffe, L.G.: Finite temperature $S U(2)$ lattice gauge theory. Commun. Math. Phys. 100, 313 (1985)

3. Borgs, C.: Area law for spatial Wilson loops in high-temperature lattice gauge theories. Nucl. Phys. B 261, 455 (1985)

4. Tomboulis, E.T., Yaffe, L.G.: Chiral symmetrie restoration at finite temperature. Phys. Rev. Lett. 52, 2115 (1984)

5. Kogut, J., Susskind, L.: Hamiltonian formulation of Wilson's lattice gauge theories. Phys. Rev. D 11, 395 (1975)

6. Osterwalder, K., Seiler, E.: Gauge field theories on a lattice. Ann. Phys. 110, 440 (1978)

7. Seiler, E.: Gauge theories as a problem of constructive quantum field theory and statistical mechanics. Lecture Notes in Physics, Vol. 159. Berlin, Heidelberg, New York: Springer 1982

8. Svetitski, B., Yaffe, L.G.: Critical behaviour at finite temperature confinement transitions. Nucl. Phys. B 210 [FS6], 423 (1982)

9. Polyakov, A.: Thermal properties of gauge fields and quark liberation. Phys. Lett. 72 B, 477 (1978)

10. Ginibre, J.: Existence of phase transitions for quantum lattice systems. Commun. Math. Phys. 14, 205 (1969)

11. Gruber, C., Kunz, H.: General properties of polymer systems. Commun. Math. Phys. 22, 133 (1971)

12. Hugenhotz, N.M.: States and representations in statistical mechanics. In: Mathematics of contempory physics. Streater, R.F. (ed.). New York, London: Academic Press 1972

13. Glimm, J., Jaffe, A.: The $\lambda\left(\phi^{4}\right)_{3}$ quantum field theory without cutoffs. III. The physical vacuum. Acta Math. 125, 203 (1970)

14. Simon, B., Yaffe, L.G.: Rigorous perimeter law upper bound on Wilson loops. Phys. Lett. $115 \mathrm{~B}, 145$ (1982)

15. Reed, M., Simon, B.: Methods of modern mathematical physics, Vol. 2. New York, London: Academic Press 1975

16. Seiler, E., Simon, B.: Nclson's symmetry and all that in the Yukawa and $\left(\phi^{4}\right)_{3}$ theories. Ann. Phys. 97, 470 (1976)

17. Brydges, D.: A short course on cluster expansions. In: Critical phenomena, random systems, gauge theories. Osterwalder, K., Stora, R. (eds.). Les Houches 1984. Amsterdam: NorthHolland 1986

18. Borgs, C.: Zufallsflächen und Clusterentwicklungen in Gitter-Yang-Mills Theorien. Thesis, University of Munich 1986

19. Mack, G.: Nonperturbative methods. In: Gauge theories of the eighties. Raitio, R., Lindfors, J. (eds.). Lecture Notes in Physics, Vol. 181. Berlin, Heidelberg, New York: Springer 1983

20. Nill, F.: Untersuchungen zur Phasenstruktur abel'scher Higgsmodelle in der Gittereichtheorie. Thesis, University of Munich 1987

21. Behnke, H., Sommer, F.: Theorie der analytischen Funktionen einer Veränderlichen. Grundlehren der math. Wissenschaften, Bd. 77. Berlin, Heidelberg, New York: Springer 1965

22. Borgs, C.: Charged surfaces and the analyticity properties of the string tension in lattice gauge theories. Trebon 1986. J. Stat. Phys. 47, 867 (1987)

23. Bricmont, J., Fröhlich, J.: Statistical mechanical methods in particle structure analysis of lattice gauge field theories I-III. Nucl. Phys. B 251 [FS 13], 517 (1985); Commun. Math. Phys. 98, 553 (1985); Nucl. Phys. B 280 [FS 18], 385 (1987)

Communicated by K. Gawedzki

Received August 24, 1987 